\title{
Variaciones espacio-temporales del nivel y salinidad del agua afectan la composición de especies del manglar-tular
}

\section{Spatial-temporal variations in water level and salinity affect species}

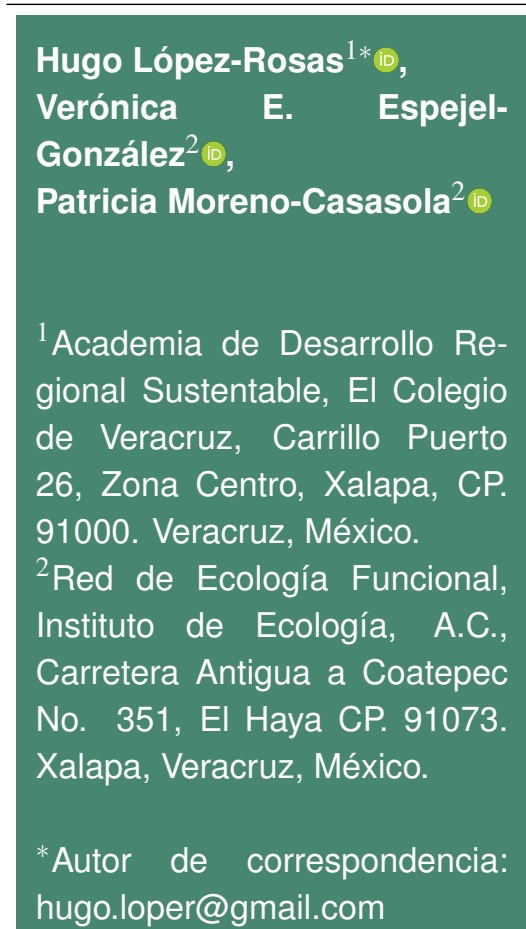

Artículo científico

Recibido: 07 de julio 2020

Aceptado: 13 de mayo 2021

Como citar: López-Rosas H, Espejel-González VE, MorenoCasasola P (2021) Variaciones espacio-temporales del nivel y salinidad del agua afectan la composición de especies del manglar-tular. Ecosistemas y Recursos Agropecuarios Núm. Esp. I: e2674. DOI: 10.19136/era.a8nl.2674
RESUMEN. La ubicación de los manglares ribereños vinculada al constante aporte de agua dulce favorece su productividad y diversidad, así como a los principales humedales dulceacuícolas adyacentes (selvas inundables, popales, tulares). Por lo mismo, la conectividad hidrológica entre los manglares y humedales adyacentes es un aspecto importante para determinar el grado de influencia entre ecosistemas y los posibles impactos al manglar por las afectaciones a otros humedales con los que interactúa. En este estudio se monitoreó, a mediano plazo (2015-2016 y 2018-2019), la composición y estructura de la vegetación y se caracterizó la conectividad hidrológica entre el manglar ribereño y humedales de agua dulce adyacentes en 27 unidades de monitoreo (22 en manglar, cinco en humedal). El nivel del agua aumentó gradualmente conforme aumentó la distancia al río, mientras que lo contrario ocurrió con la salinidad. En el periodo de 2018-2019 la salinidad del agua intersticial aumentó en promedio 10 UPS, lo que provocó cambios en la presencia y cobertura de algunas especies como Laguncularia racemosa en la zona del tular, alta mortandad de especies herbáceas (Acrostichum danaeifolium, Typha domingensis, Phragmites australis) y especies arbóreas como Annona glabra y Acoelorraphe wrightii, entre las más evidentes. Sin embargo, no se detectaron cambios estructurales significativos en las zonas dominadas por manglar. En conclusión, la vegetación respondió a un gradiente de nivel del agua y salinidad del agua intersticial, ya que cerca del río se presentaron niveles bajos de agua y altos de salinidad y lo contrario ocurrió tierra adentro.

Palabras clave: Conectividad, gradiente, México, Reserva de la Biósfera Pantanos de Centla, salinización, zona costera.

ABSTRACT. The location of the riverine mangroves linked to the constant supply of freshwater favors their productivity and diversity, as well as the main adjacent freshwater wetlands (freshwater swamps, broadleaved marshes, cattail marshes). For this reason, the hydrological connectivity between the mangroves and adjacent wetlands is an important aspect that determines the degree of influence between ecosystems and the possible impacts to the mangrove derived from the effects on other wetlands with which it interacts. In this study, the composition and structure of the vegetation were monitored in the medium term (2015-2016 and 2018-2019) and the hydrological connectivity between the riparian mangrove and adjacent freshwater wetlands was characterized in 27 monitoring units (22 in mangroves, five in wetlands). The water level increased gradually as the distance to the river increased, while the opposite happened with salinity. In the period 2018-2019, the salinity of the interstitial water increased by an average of $10 \mathrm{PSU}$, causing changes in the presence and cover of some species such as Laguncularia racemosa in the cattail zone, high mortality of herbaceous species (Acrostichum danaeifolium, Typha domingensis, Phragmites australis) and tree species such as Annona glabra and Acoelorraphe wrightii, among the most evident. However, no significant structural changes were detected in areas dominated by mangroves. In conclusion, the vegetation responded to a gradient in the water level and salinity of the interstitial water since near the river were registered low levels of water and high salinity, while the opposite occurred inland.

Key words: Connectivity, coastal zone, gradient, Mexico, Pantanos de Centla Biosphere Reserve, salinization. 


\section{INTRODUCCIÓN}

Los manglares ribereños son comunidades arbóreas asociadas a los bordes de los ríos, por lo que están influidos en mayor o menor grado por la salinidad y el nivel de marea del agua del mar (Novelo y Ramos 2005). En este tipo de manglar hay menor estrés salino, lo que permite que los manglares ribereños sean los más productivos y alcancen las mayores alturas (Cintrón et al. 1985, Herrera-Silveira et al. 2016). Este mayor aporte de agua dulce permite que los manglares ribereños tengan mayor diversidad vegetal que los demás tipos fisonómicos de manglares, por lo que en México es común encontrar a las especies de manglar (Rhizophora mangle, Avicennia germinans, Laguncularia racemosa) asociadas con especies menos tolerantes a la salinidad, como Conocarpus erectus, Annona glabra, Pachira aquatica, Acrostichum danaeifolium y distintas especies de palmas como Acoelorraphe wrightii, Roystonea dunlapiana, Attalea butyracea y Sabal spp. (Olmsted 1993, González-Marín et al. 2012, Infante-Mata et al. 2014).

Los humedales ubicados en las planicies costeras, tanto selvas inundables como popales y tulares, son los ecosistemas más vulnerables al aumento en el nivel del mar por el cambio climático actual (Craft et al. 2009, Zhang et al. 2019). En ellos los cambios en su dinamismo, están dados por el hidroperíodo, definido por la frecuencia, periodicidad y nivel de inundación, las fluctuaciones de la marea (la salinidad del agua), los aportes de agua dulce de los ríos, la precipitación y la fluctuación del agua subterránea (Barendregt y Swarth 2013). Diversos autores (Herbert et al. 2015, McCarthy et al. 2018, Zhai et al. 2018, van Dij et al. 2019, Wilson et al. 2019) han demostrado la afectación por la intrusión salina a causa del aumento del nivel del mar en diferentes regiones costeras del mundo. Otra causa no menos importante son las modificaciones a los flujos en las cuencas, ya que es un factor crítico para entender el origen y la calidad del agua que por esta vía alimenta al humedal, así como la interacción entre los diferentes responsables de la dinámica con el ecosistema (Sánchez-Higueredo et al. 2020).
Los humedales de agua dulce adyacentes a los manglares ribereños se caracterizan por concentraciones bajas de salinidad en suelo y agua, las cuales favorecen la distribución de las selvas inundables (p. ej. de Pachira aquatica, Annona glabra, Astianthus viminalis; Olmsted 1993), los tulares (de Typha domingensis o Cyperus giganteus), los carrizales (Phragmites communis), los matorrales (Mimosa pigra, Dalbergia glabra, D. brownei), las sabanas inundables (mezcla de especies graminoides como Paspalum sp. o Aristida sp. con árboles aislados de Byrsonima crassifolia, Curatella americana, Crescentia alata o C. cujete entre otros), los popales (Pontederia sagittata, Thalia geniculata), o los terrenos manejados por el hombre, como pastizales inundables para potreros y caña de azúcar (Olmsted 1993). El tipo de ecosistema asociado al manglar ribereño está determinado por las características hidrológicas y del relieve de cada sitio en particular (Flores-Verdugo et al. 2007). Mientras que el grado de conectividad hidrológica lateral, entre el manglar y este tipo de ecosistemas determina, entre otros aspectos, los cambios que se pueden producir en los ecosistemas colindantes (Bracken y Croke 2007). Ambos tipos de humedales costeros son afectados por pulsos de inundación, condiciones hidrometeorológicas como las derivadas del aumento rápido del nivel del río por lluvias o por marejadas de tormenta, en los que en corto tiempo hay una fuerte entrada de sedimentos ricos en nutrientes y sales al sistema (Nyman et al. 1990, Day et al. 1995).

Por su ubicación en la cuenca, los manglares ribereños no están asociados a pastos marinos, ni a arrecifes, pero sí a humedales de agua dulce de distintos tipos (Davis et al. 2005). La conectividad hidrológica entre manglares y humedales de agua dulce, así como su importancia en la generación de bienes y servicios ecosistémicos, ha sido documentada por Ewel (2010) y por Davis et al. (2005). Con el propósito de entender la conectividad hidrológica entre manglares ribereños y humedales de agua dulce, el presente trabajo está enfocado a evaluar cambios graduales, tanto espaciales como temporales, en la composición y estructura de la vegetación, nivel del agua y otras características fisicoquímicas 
de suelo y agua, como $\mathrm{pH}$, salinidad, Eh, densidad aparente y contenido de agua en suelo. El nivel de inundación, su periodicidad y estacionalidad constituyen el hidroperíodo, llamado la firma del humedal por Mitsch y Gosselink (2007), ya que son característicos de cada tipo de humedal. Por lo mismo, el hidroperíodo y la fluctuación de las variables ambientales que miden los niveles de agua y su salinidad ayudan a explicar la distribución de la flora y fauna de los humedales. Entre estas variables, el potencial de óxido-reducción disminuye cuando aumenta la profundidad o el tiempo de inundación; la densidad aparente en zonas de inundación más prolongadas disminuye junto con la tasa de descomposición de la materia orgánica del suelo, y la salinización del agua dulce frecuentemente ocurre con otros cambios en el pH (Zalisniak et al. 2009). Por tanto, las fluctuaciones en los niveles de inundación y en la salinidad están relacionados con diversas variables ambientales y afectan a la flora y fauna de los humedales.

Específicamente, los estudios ecológicos y de conectividad hidrológica se han enfocado a ecosistemas estuarinos y por ello la información relacionada con la influencia de humedales de agua dulce sobre manglares y viceversa es muy escasa (LotHelgueras y Novelo 1990, López-Portillo y Ezcurra 2002, Ellison 2004, McKee 2012, Moreno-Casasola et al. 2012). Esta carencia de estudios sobre humedales costeros de agua dulce se refleja en las estrategias de conservación en la zona costera, donde se prioriza la protección de manglares, pero se descuida lo que ocurre en el margen posterior de los manglares, que es donde se encuentran los humedales de agua dulce y donde está ocurriendo la mayor pérdida de humedales por cambios de uso del suelo (Moreno-Casasola 2008, Landgrave y MorenoCasasola 2012). Sólo por mencionar un ejemplo, el polígono del área protegida de flora y fauna Laguna de Términos está delimitado por la presencia de manglares, por lo que las únicas zonas protegidas y conservadas son las que contienen manglares, mientras que los humedales de agua dulce, de los que dependen hidrológicamente los manglares, ahora están transformados en potreros, arrozales (Benítez Torres y Villalobos-Zapata 2010), o más recientemente en cultivos de palma de aceite (Rosas Urióstegui et al. 2018).

Los objetivos generales que se plantearon en esta investigación fueron determinar en el mediano plazo: a) la composición y estructura de la vegetación a lo largo de un gradiente ambiental en agua intersticial, agua superficial y suelo, y b) la conectividad hidrológica entre el manglar y los humedales de agua dulce. Los objetivos particulares que se establecieron fueron: i) determinar las especies de plantas y monitorear los cambios y las características espaciales de la vegetación con respecto a la incidencia estacional de las variables del sustrato y del agua en el manglar y el humedal de agua dulce, ii) medir cada 30 días la fluctuación temporal de las variables asociadas a la inundación, así como el potencial de óxido reducción, densidad aparente y $\mathrm{pH}$, iii) monitorear cada 30 días las variaciones temporales-estacionales de la fluctuación del incremento o descenso del nivel y salinidad del agua en el manglar y en el humedal, (iv) determinar el grado de conectividad hidrológica y su dinámica entre el manglar y el humedal de agua dulce y finalmente (v) determinar los indicadores de cambio en la conectividad entre ambos ecosistemas.

Las hipótesis planteadas consideraron que las variaciones en la salinidad y el nivel del agua ( $y$ variables asociadas a la inundación, como potencial de óxido reducción, densidad aparente y $\mathrm{pH}$ ), traerán cambios tanto en la estructura como en la composición de la vegetación en los manglares y humedales de agua dulce. Una primera hipótesis plantea que un incremento en salinidad en los manglares llevaría a reducir la presencia de especies menos tolerantes como $L$. racemosa e incrementar la de especies más tolerantes, $R$. mangle y $A$. germinans. En el caso de los humedales herbáceos de agua dulce, se puede producir mortandad de individuos y la posterior colonización por especies más tolerantes a la salinidad o, en caso de salinidad extrema, la formación de una marisma sin vegetación emergente. Esto mismo ocurriría para la segunda hipótesis, ya que, en el caso de cambios en el nivel de inundación, al reducirse el tiempo de inundación 
y nivel en los humedales herbáceos, se favorece la entrada de especies arbóreas; por el contrario, un aumento desplazaría a hidrófitas facultativas, como diferentes especies de gramíneas, y aumentaría la dominancia de especies como Typha domingensis.

\section{MATERIALES Y MÉTODOS}

\section{Área de estudio}

El área de estudio se ubica $\left(18^{\circ} 37^{\prime} 50.45^{\prime \prime} \mathrm{N}\right.$, $92^{\circ} 29^{\prime} 44.85^{\prime \prime} \mathrm{O}$ y $18^{\circ} 36^{\prime} 48.90^{\prime \prime} \mathrm{N} 92^{\circ} 28^{\prime} 02.45^{\prime \prime}$ O) en la vertiente poniente del Río San Pedro y San Pablo a $1.3 \mathrm{~km}$ de su desembocadura en el sur del Golfo de México (Figura 1 A). El área se encuentra en la zona de amortiguamiento de la Reserva de la Biósfera Pantanos de Centla (Guerra-Martínez y Ochoa-Gaona 2018). Su clima es cálido subhúmedo; la mayor precipitación ocurre principalmente en octubre y noviembre (Figura $1 \mathrm{C}$ ); la temperatura media anual fue de $25.9{ }^{\circ} \mathrm{C}$ y la precipitación media anual en años secos ha fluctuado de 1370 a 1420 mm; mientras que en años lluviosos ha variado entre 1760 y $1800 \mathrm{~mm}$ (Novelo-Retana 2006). Por el volumen de precipitación pluvial y la intensidad de los vientos generados por los frentes fríos de invierno, las estaciones climáticas en la zona se clasifican en "secas" (de febrero a mayo), "lluvias" (de junio a septiembre) y "nortes" (de octubre a febrero) (Rivera-Monroy et al. 2011).

\section{Método de muestreo de la estructura de la vegetación}

El diseño de muestreo seleccionado fue de tipo sistemático (Ratti y Garton 1996). Este diseño permite detectar la variación espacio-temporal de características bióticas y abióticas de un área, y puede ser aplicado para manglar y humedal. El procedimiento consistió en ubicar tres transectos con orientación Oriente-Poniente partiendo del borde del río (manglar ripario) hacia el humedal herbáceo (tular) y con $150 \mathrm{~m}$ de separación entre transectos contiguos. A lo largo de cada transecto, cada $250 \mathrm{~m}$ se ubicaron nueve unidades permanentes de muestreo denominadas UM (en el transecto central hubo ocho UM con vegetación arbórea y una con vegetación herbácea; en los transectos laterales hubo siete UM con vegetación arbórea y dos con vegetación herbácea en cada uno). Uno de los objetivos particulares fue detectar los cambios espaciales graduales de la vegetación, y por ello los transectos terminaron en los primeros 500 metros después de la desaparición del manglar y su reemplazo por vegetación herbácea. No se consideró hacer transectos más largos que permitieran aumentar el número de muestras de vegetación herbácea, porque se llegó a un tular de características homogéneas en hidrología y composición de la vegetación.

Las unidades de muestreo (UM) fueron cuadros de $10 \times 10 \mathrm{~m}\left(100 \mathrm{~m}^{2}\right)$, con dos subcuadros de $4 \times 4 \mathrm{~m}\left(16 \mathrm{~m}^{2}\right)$ y cuatro sub-subcuadros de $1 \times 1$ $\mathrm{m}\left(1 \mathrm{~m}^{2}\right)$, de acuerdo con la metodología propuesta por Valdez Hernández (2002) (Figura 1 B). En los cuadros de $10 \times 10 \mathrm{~m}$ se midió el diámetro a la altura del pecho (DAP, con cinta diamétrica marca Forestry Suppliers) y la altura de los árboles adultos (mayores a $2.5 \mathrm{~cm}$ de DAP). Para medir la altura total del árbol se usó un clinómetro marca Brunton (CM36OLA) y una cinta métrica de fibra de vidrio de $50 \mathrm{~m}$, siguiendo el procedimiento estándar descrito por Brower et al. (1998). En los cuadros de $4 \times 4 \mathrm{~m}$ se estimó la cobertura en escala porcentual (Kent, 2011) y la altura promedio de cada especie, considerando únicamente a los individuos juveniles (menores de $2.5 \mathrm{~cm}$ de DAP y mayores de $30 \mathrm{~cm}$ de altura) y a las especies arbustivas. En los cuadros de $1 \times 1 \mathrm{~m}$ se estimó la cobertura porcentual y la altura promedio de cada especie, considerando únicamente las plántulas (menores de $30 \mathrm{~cm}$ de altura) y a las especies herbáceas. Los muestreos de la vegetación se hicieron en mayo de 2015 y octubre de 2019.

\section{Valor de Importancia Relativa (VIR) de las es- pecies vegetales}

Un VIR para cada especie se obtuvo en cada UM. Para las especies arbóreas el VIR se obtuvo con la siguiente fórmula $\mathrm{VIR}_{\text {árboles }}=$ (Área basal relativa + altura relativa) /2; mientras que para las especies herbáceas y arbustivas el VIR se estimó con $\mathrm{VIR}_{\text {hierbasyarbustos }}=($ Cobertura relativa + Altura relativa) $/ 2$. 


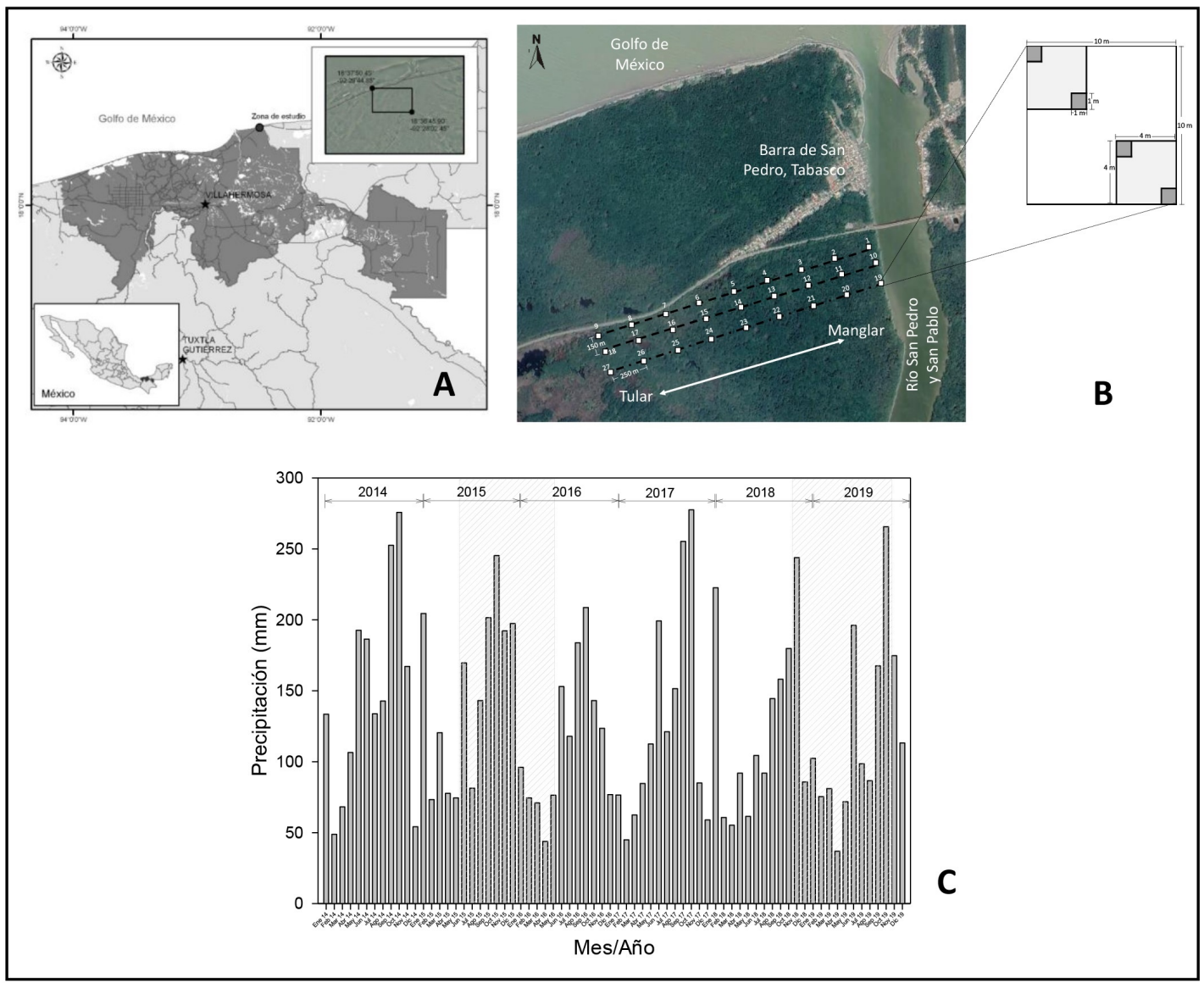

Figura 1. A) Ubicación del área de estudio en el Río San Pedro y San Pablo, Centla, Tabasco. B) Esquema del diseño de muestreo de 27 Unidades de Monitoreo (UM) distribuidas sistemáticamente en tres transectos en dirección Oriente-Poniente, y del cuadro y subcuadros de cada UM para el monitoreo de la composición y estructura de la vegetación. C) Gráfica de la precipitación mensual en el área de estudio entre 2014 y 2019 con datos de la Comisión Nacional del Agua (CONAGUA 2021); el periodo de junio 2014 a mayo 2016 fue afectado por el fenómeno El Niño (Yin et al. 2021) y tuvo una precipitación acumulada de $3338 \mathrm{~mm}$; el periodo de noviembre 2017 a octubre 2019 fue más seco, y tuvo $2825 \mathrm{~mm}$ ( $513 \mathrm{~mm}$ menos que el primer periodo). El sombreado indica los meses en que se ejecutó el presente estudio.

El área basal relativa de cada especie arbórea se obtuvo sumando las áreas basales $\left(\mathrm{en}^{2}\right.$ ) de los individuos de la misma especie en la UM y dividiendo entre 100. La cobertura relativa se obtuvo dividiendo el valor de la cobertura porcentual de cada especie herbácea o arbustiva en la UM entre 100. La altura relativa se obtuvo dividiendo la altura promedio de la especie en la UM y dividiendo entre la altura máxima obtenida en el total de los muestreos, que fue de 16.4 $\mathrm{m}$.

\section{Características fisicoquímicas de agua y suelo}

En la parte central de cada UM se instaló un piezómetro de PVC de $1.25 \mathrm{~cm}$ de diámetro a una profundidad de $50 \mathrm{~cm}$ acorde con los lineamientos de Peralta Peláez et al. (2009) para medir mensualmente el nivel del agua y obtener muestras de agua intersticial. Las muestras de agua y suelo se obtuvieron cada 30 días en dos periodos; a) de junio de 2015 a mayo de 2016, y b) de noviembre de 2018 a octubre de 2019. El pH, Eh, temperatura y salinidad se midieron in situ en todas las muestras de agua intersticial con un equipo multiparamétrico (Ultrameter II Mod. 6PFC, Myron L). Como referencia de la salinidad del agua, en cada evento de muestreo también se midió la salinidad 
del agua superficial del río en la parte más cercana del inicio de cada transecto. Para evaluar las condiciones de oxidación-reducción a que están expuestas las raíces, según López Rosas y Tolome Romero (2009), contiguo a cada piezómetro, se instalaron tres electrodos de platino (a una profundidad de $30 \mathrm{~cm}$ del suelo). En cada evento de muestreo se insertó un electrodo cálomel de referencia (Fisher Scientific 13620-259) entre los tres electrodos de platino. El electrodo cálomel se conectó a la salida negativa (tierra) de un voltímetro (Steren mul-010), mientras que a la salida positiva se conectaron consecutivamente cada uno de los electrodos de platino, de tal forma que para una misma UM se tienen tres valores del potencial de óxido-reducción (ORP) del suelo. El valor promedio de las tres lecturas se corrigió añadiendo $244.3 \mathrm{mV}$ para obtener el valor de Eh del suelo (López Rosas y Tolome Romero 2009).

En cada UM se recolectaron muestras de suelo de volumen conocido $\left(176.7 \mathrm{~cm}^{3}\right)$ con un cilindro de PVC en los primeros $10 \mathrm{~cm}$ de suelo. De esta muestra se obtuvo su peso húmedo. Posteriormente, la muestra de suelo se secó a $65^{\circ} \mathrm{C}$ hasta obtener un peso constante y se volvieron a pesar (peso seco). La densidad aparente se calculó dividiendo el peso seco $(\mathrm{g})$ entre $176.7 \mathrm{~cm}^{3}$. El contenido porcentual de agua en suelo se obtuvo con la siguiente fórmula: Contenido de agua $(\%)=$ (peso húmedo - peso seco) / peso húmedo x 100.

\section{Análisis de datos}

Los valores obtenidos de densidad aparente, área basal y altura de cada especie, así como la densidad total, área basal total y altura promedio general, en el muestreo de mayo del 2015 se compararon con los obtenidos en octubre de 2019. Cuando las variables pasaron los supuestos de normalidad y equitabilidad, la comparación estadística se hizo con una prueba $t$ de Student (Zar 2009); cuando las variables no pasaron los supuestos, la comparación se hizo con una prueba $U$ de Mann-Whitney (Zar 2009). Los valores obtenidos en el periodo 2015-2016 de las variables fisicoquímicas de suelo y agua se compararon con los obtenidos en el periodo 2018-2019 con una prueba de t pareada (Zar
2009). Previo a la ejecución del análisis, los valores porcentuales de contenido de agua en suelo se transformaron con el arco seno de la raíz cuadrada para cumplir los supuestos de normalidad y equitabilidad. Los VIR de cada especie se integraron en una matriz de datos con 54 UM y 26 tipos funcionales de 16 morfoespecies (como Rhizophora mangle adulto, $R$. mangle juvenil y $R$. mangle plántula). Estos datos se transformaron elevando al cuadrado y después se analizaron por medio de matrices de similitud Bray-Curtis, con las que se formaron grupos (análisis CLUSTER; Legendre y Legendre 1998). Para detectar diferencias entre grupos de UM se aplicó un análisis del porcentaje de la similitud (SIMPROF, Clarke et al. 2008) por medio de métodos de permutación y aleatorización de la matriz de similitudes. Para determinar las especies determinantes de cada grupo formado, los grupos con significancia estadística obtenidos del SIMPROF se describieron con el apoyo de la técnica de análisis de similitud entre porcentajes y contribución de especies (SIMPER, Clarke 1993). Los datos abióticos se integraron en otra matriz, se estandarizaron y posteriormente se usaron para un análisis de gradiente indirecto (BEST/BIOENV, Clarke et al. 2008) para interpretar las clasificaciones en relación con las variables ambientales. Para verificar la conectividad hidrológica entre los principales tipos de vegetación, se graficó y comparó (con prueba de t pareada entre meses) el nivel del agua y la salinidad intersticial de seis UM del extremo oriente (cercano al río) con seis UM del extremo poniente (hacia tierra firme). Las pruebas de $t$ y $U$ se hicieron en el software SigmaPlot ver. 11.0; los análisis CLUSTER, SIMPROF, SIMPER y BEST/BIOENV se hicieron con PRIMER 6.0; la ordenación MDS se hizo con PCORD 6.

\section{RESULTADOS}

\section{Caracterización fisonómica de la vegetación}

El área de estudio presentó un gradiente de la fisonomía de la vegetación en dirección OrientePoniente (del borde del río hacia tierra firme). En dirección Norte-Sur no hubo un gradiente detectable. La caracterización fisonómica de la vegetación a lo 
largo del gradiente de inundación muestra que las UM cercanas al río tuvieron densidades bajas de árboles altos (de hasta $16 \mathrm{~m}$ ) y con diámetros de hasta 40 $\mathrm{cm}$. En esta sección las especies dominantes fueron $R$. mangle y $L$. racemosa, con poca presencia de $A$. germinans. Conforme se incrementó la distancia con respecto a la orilla del río, las UM registraron árboles con densidades altas, pero estuvieron dominadas por individuos arbustivos (diámetros menores de 17 $\mathrm{cm}$ ) de $L$. racemosa con alturas menores de nueve metros y parches de vegetación herbácea dominada por Acrostichum danaeifolium, así como escasos individuos de $A$. glabra. En la sección más alejada del río, la vegetación arbóreo-arbustiva fue escasa e inició la dominancia por vegetación herbácea con especies como Phragmites australis y Typha domingensis, con parches de la palma Acoelorraphe wrigthii y de $L$. racemosa, ésta última ya creciendo con forma de arbusto; o bien matorrales dominados por el arbusto Dalbergia brownei, creciendo junto con $P$. australis.

\section{Estructura de la vegetación}

En 22 de las 27 UM dominó vegetación arbórea compuesta por especies de mangle. En las otras cinco UM del gradiente muestreado predominó la vegetación herbácea característica de humedales de agua dulce. La especie más abundante fue $L$. racemosa, aunque la distribución espacial de esta especie de mangle a lo largo del gradiente mostró diferencias estructurales, ya que presentó mayores alturas y menores densidades al inicio del gradiente $\left(10.7 \pm 1.0 \mathrm{~m} ; 358.3 \pm 96.4\right.$ ind $\cdot \mathrm{ha}^{-1}$, pero mayores densidades con menores alturas en la parte central $\left(5.9 \pm 0.3 \mathrm{~m} ; 4000.0 \pm 1082.6 \mathrm{ind} \cdot \mathrm{ha}^{-1}\right)$. En la Tabla 1 se presentan los VIR de las especies a lo largo del gradiente, es decir las características de la distribución espacial. La segunda especie en dominancia fue $R$. mangle, y su abundancia se fue reduciendo. En las UM ubicadas al final del gradiente se registró $A$. germinans, especie que sólo estuvo presente en las UM 8, 9, 17 y 18. Las características estructurales de ambos muestreos, es decir de la distribución temporal, resultaron estadísticamente similares entre las comparaciones a nivel de especie y de comunidad
(Tabla 2).

\section{Características fisicoquímicas de suelo y agua}

La comparación estadística de cada variable fisicoquímica medida entre los dos periodos se presenta en la Tabla 3. Sólo los valores de salinidad intersticial y de salinidad superficial del río aumentaron significativamente en el periodo 2018-2019, en relación al periodo 2015-2016.

La Figura 2 muestra las fluctuaciones del nivel del agua y de la salinidad del agua intersticial a lo largo del gradiente en las épocas de secas (diciembre-mayo) y lluvias (junio-noviembre). El manglar de borde presentó los niveles más bajos de agua y el tular los más altos, tanto en secas como en lluvias. También que los niveles de agua fueron más altos en la temporada de secas que en la de lluvias. La salinidad intersticial resultó más alta en las zonas donde se distribuyó $R$. mangle, intermedia en las zonas de $L$. racemosa y menor en los tulares. La salinidad del agua intersticial registró valores más altos en la época de lluvias, es decir cuando el nivel del agua es más bajo (Figura 2 ).

Los resultados de la variación mensual del nivel del agua (Figura $3 \mathrm{~A}$ ), de la salinidad del agua intersticial de las 27 UM (Figura 3 B) y de la salinidad del agua superficial del río (Figura $3 \mathrm{C}$ ) para los dos periodos de estudio, 2015-2016 y 2018-2019, indicaron que entre ambos periodos hubo un incremento significativo de la salinidad del agua intersticial de las UM y del agua superficial del río (de 10 a 15 UPS) en el periodo 2015-2016 con respecto al periodo 20182019. El primer periodo coincidió con el fenómeno de El Niño (Yin et al. 2021), que en el 2014-2015 registró una sequía extrema, seguida de abundante lluvia del 2015 al 2016 (ver climograma en la Figura 1). En contraste, en el periodo 2018-2019 la precipitación acumulada de 2017-2019 resultó $513 \mathrm{~mm}$ menor que la del periodo de El Niño de 2014-2016 (Figura 1). También se observó un incremento en el nivel del agua entre ambos periodos, aunque éste no fue estadísticamente significativo $(P=0.47$; Tabla 3$)$. En ambos periodos la salinidad del agua intersticial fue menor en la época de secas (Figura 3 B). La salinidad del agua superficial (Figura $3 \mathrm{C}$ ) mostró un fuerte incre- 
Tabla 1. Valores de importancia relativa de las principales especies en relación con la distancia al río. Se presenta el promedio de tres Unidades de Monitoreo (UM) \pm 1 error estándar.

\begin{tabular}{|c|c|c|c|c|c|c|c|}
\hline \multicolumn{8}{|l|}{2015} \\
\hline $\begin{array}{l}\text { Distancia } \\
\text { al río }(\mathrm{m})\end{array}$ & $\begin{array}{l}\text { Laguncularia } \\
\text { racemosa }\end{array}$ & $\begin{array}{l}\text { Rhizophora } \\
\text { mangle }\end{array}$ & $\begin{array}{l}\text { Avicennia } \\
\text { germinans }\end{array}$ & $\begin{array}{l}\text { Typha domin- } \\
\text { gensis }\end{array}$ & Setaria sp. & $\begin{array}{l}\text { Phragmites } \\
\text { australis }\end{array}$ & $\begin{array}{l}\text { Dalbergia } \\
\text { brownei }\end{array}$ \\
\hline 53.1 & $0.19 \pm 0.10$ & $0.14 \pm 0.14$ & $0.06 \pm 0.02$ & 0 & 0 & 0 & 0 \\
\hline 303.6 & $0.25 \pm 0.16$ & $0.28 \pm 0.28$ & $0.27 \pm 0.32$ & 0 & 0 & 0 & 0 \\
\hline 554.8 & $0.15 \pm 0.14$ & $0.32 \pm 0.32$ & 0 & 0 & 0 & 0 & 0 \\
\hline 804.9 & $0.09 \pm 0.02$ & $0.50 \pm 0.38$ & 0 & 0 & 0 & 0 & 0 \\
\hline 1138.9 & $0.50 \pm 0.40$ & $0.15 \pm 0.16$ & 0 & 0 & 0 & 0 & 0 \\
\hline 1388.7 & $0.32 \pm 0.08$ & $0.02 \pm 0.03$ & 0 & 0 & 0 & 0 & 0 \\
\hline 1639.0 & $0.19 \pm 0.12$ & $0.03 \pm 0.06$ & 0 & 0 & 0 & 0 & $0.03 \pm 0.05$ \\
\hline 1889.4 & $0.16 \pm 0.27$ & 0 & 0 & $0.10 \pm 0.18$ & $0.17 \pm 0.29$ & $0.02 \pm 0.04$ & $0.03 \pm 0.04$ \\
\hline 2139.3 & 0 & 0 & 0 & $0.23 \pm 0.08$ & 0 & $0.14 \pm 0.14$ & $0.09 \pm 0.15$ \\
\hline \multicolumn{8}{|l|}{2019} \\
\hline $\begin{array}{l}\text { Distancia } \\
\text { al río (m) }\end{array}$ & $\begin{array}{l}\text { Laguncularia } \\
\text { racemosa }\end{array}$ & $\begin{array}{l}\text { Rhizophora } \\
\text { mangle }\end{array}$ & $\begin{array}{l}\text { Avicennia } \\
\text { germinans }\end{array}$ & $\begin{array}{l}\text { Typha domin- } \\
\text { gensis }\end{array}$ & Setaria sp. & $\begin{array}{l}\text { Phragmites } \\
\text { australis }\end{array}$ & $\begin{array}{l}\text { Dalbergia } \\
\text { brownei }\end{array}$ \\
\hline 53.1 & $0.18 \pm 0.08$ & $0.11 \pm 0.15$ & $0.05 \pm 0.02$ & 0 & 0 & 0 & 0 \\
\hline 303.6 & $0.18 \pm 0.11$ & $0.20 \pm 0.11$ & $0.13 \pm 0.15$ & 0 & 0 & 0 & 0 \\
\hline 554.8 & $0.10 \pm 0.09$ & $0.29 \pm 0.29$ & 0 & 0 & 0 & 0 & 0 \\
\hline 804.9 & $0.11 \pm 0.01$ & $0.39 \pm 0.24$ & 0 & 0 & 0 & 0 & 0 \\
\hline 1138.9 & $0.35 \pm 0.25$ & $0.13 \pm 0.12$ & 0 & 0 & 0 & 0 & 0 \\
\hline 1388.7 & $0.23 \pm 0.02$ & $0.04 \pm 0.06$ & 0 & 0 & 0 & 0 & 0 \\
\hline 1639.0 & $0.22 \pm 0.05$ & $0.04 \pm 0.07$ & 0 & 0 & 0 & 0 & $0.04 \pm 0.06$ \\
\hline 1889.4 & $0.20 \pm 0.15$ & 0 & 0 & $0.01 \pm 0.02$ & $0.17 \pm 0.30$ & 0 & $0.07 \pm 0.07$ \\
\hline 2139.3 & $0.17 \pm 0.15$ & 0 & 0 & $0.01 \pm 0.00$ & 0 & $0.03 \pm 0.04$ & $0.06 \pm 0.11$ \\
\hline
\end{tabular}

Tabla 2. Comparación entre los valores de características estructurales de la vegetación arbórea obtenidos en 22 de las 27 Unidades de Monitoreo (UM) al inicio del monitoreo (mayo 2015) y al final del monitoreo (octubre 2019). Se presenta la media $\pm 1 \mathrm{EE}$ o la mediana (1er cuartil - 3er cuartil).

\begin{tabular}{|c|c|c|c|c|c|c|}
\hline Característica & Especie & 2015 & 2019 & $t$ & $U$ & $\mathrm{P}$ \\
\hline \multirow{3}{*}{ Densidad (ind.ha ${ }^{-1}$ ) } & Avicennia germinans & $120.0 \pm 25.5 \mathrm{a}$ & $120.0 \pm 25.5 \mathrm{a}$ & 0.05 & - & 1.0 \\
\hline & Laguncularia racemosa & $\begin{array}{l}1275.0 \mathrm{a} \\
(225.0-3750.0)\end{array}$ & $\begin{array}{l}1200 \mathrm{a} \\
(175.0-3687.5)\end{array}$ & - & 9.0 & 0.78 \\
\hline & Rhizophora mangle & $\begin{array}{l}287.5 \mathrm{a} \\
(125.0-712.5)\end{array}$ & $\begin{array}{l}250.0 \mathrm{a} \\
(112.5-656.2)\end{array}$ & - & 11.0 & 0.73 \\
\hline \multirow{3}{*}{ Área basal $\left(\mathrm{m}^{2} \cdot \mathrm{ha}^{-1}\right)$} & Avicennia germinans & $\begin{array}{l}4.2 \mathrm{a} \\
(3.5-9.8)\end{array}$ & $\begin{array}{l}4.8 \mathrm{a} \\
(4.2-10.5)\end{array}$ & - & 189.0 & 0.55 \\
\hline & Laguncularia racemosa & $\begin{array}{l}11.8 \mathrm{a} \\
(5.5-24.2)\end{array}$ & $\begin{array}{l}12.6 \mathrm{a} \\
(5.9-24.4)\end{array}$ & - & 192.5 & 0.85 \\
\hline & Rhizophora mangle & $\begin{array}{l}4.6 \mathrm{a} \\
(1.8-26.8)\end{array}$ & $\begin{array}{l}4.6 \mathrm{a} \\
(1.7-19.0)\end{array}$ & - & 173.0 & 0.84 \\
\hline \multirow{3}{*}{ Altura (m) } & Avicennia germinans & $\begin{array}{l}11.4 \mathrm{a} \\
(11.0-12.3)\end{array}$ & $\begin{array}{l}11.3 \mathrm{a} \\
(11.2-12.1)\end{array}$ & - & 126.0 & 0.84 \\
\hline & Laguncularia racemosa & $\begin{array}{l}5.9 a \\
(5.5-9.3)\end{array}$ & $\begin{array}{l}6.1 \mathrm{a} \\
(5.7-10.4)\end{array}$ & - & 130.0 & 0.47 \\
\hline & Rhizophora mangle & $9.0 \pm 0.4 \mathrm{a}$ & $8.2 \pm 0.5 \mathrm{a}$ & 1.2 & - & 0.22 \\
\hline Densidad total (ind.ha ${ }^{-1}$ ) & & $\begin{array}{l}1212.5 \mathrm{a} \\
(675.0-4200.0)\end{array}$ & $\begin{array}{l}1300.0 \mathrm{a} \\
(706.2-3343.7)\end{array}$ & - & 247.5 & 0.91 \\
\hline Área basal total $\left(\mathrm{m}^{2} \cdot \mathrm{ha}^{-1}\right)$ & & $\begin{array}{l}25.2 \mathrm{a} \\
(11.3-33 .)\end{array}$ & $\begin{array}{l}23.8 \mathrm{a} \\
(12.5-36.4)\end{array}$ & - & 235.0 & 0.69 \\
\hline Altura $(m)$ & & $\begin{array}{l}7.0 \mathrm{a} \\
(5.9-9.9)\end{array}$ & $\begin{array}{l}7.0 \mathrm{a} \\
(6.1-10.3)\end{array}$ & - & 246.0 & 0.88 \\
\hline
\end{tabular}

mento durante las secas; la mayor variación temporal se detectó en el segundo periodo con un recorrido de 32.2 UPS (de 1.4 a 33.6 UPS); mientras que en el primer periodo el recorrido fue de 20.3 UPS (de 0.4 a 20.7 UPS). En ambos periodos de estudio el nivel del agua fue mayor al inicio de la temporada de secas (Figura $3 \mathrm{~A}$ ) y fue disminuyendo gradualmente hasta alcanzar sus valores más bajos a finales de secas y principios de lluvias (mayo-junio). En los primeros meses de lluvias (junio-septiembre) el nivel del agua 
Tabla 3. Comparación de las características fisicoquímicas de suelo y agua entre los valores obtenidos en los periodos 2015-2016 y 2018-2019. Se presenta la media $\pm 1 \mathrm{EE}$.

\begin{tabular}{lllll}
\hline Característica & $2015-2016$ & $2018-2019$ & $t_{\text {pareada }}$ & $\mathrm{P}$ \\
\hline Nivel del agua (cm) & $2.63 \pm 9.34 \mathrm{a}$ & $9.29 \pm 4.59 \mathrm{a}$ & 0.74 & 0.47 \\
Salinidad del agua intersticial (UPS) & $21.77 \pm 1.23 \mathrm{~b}$ & $31.87 \pm 1.31 \mathrm{a}$ & 6.02 & $<0.001$ \\
Salinidad del agua superficial del río (UPS) & $3.87 \pm 1.00 \mathrm{~b}$ & $19.10 \pm 2.83 \mathrm{a}$ & 5.50 & $<0.001$ \\
ph del agua intersticial & $7.38 \pm 0.11 \mathrm{a}$ & $7.13 \pm 0.07 \mathrm{a}$ & 1.73 & 0.11 \\
Temperatura del agua intersticial $\left({ }^{\circ} \mathrm{C}\right)$ & $26.76 \pm 0.49 \mathrm{a}$ & $28.04 \pm 0.59 \mathrm{a}$ & 1.91 & 0.08 \\
Eh del agua intersticial $(\mathrm{mV})$ & $22.50 \pm 12.60 \mathrm{a}$ & $39.34 \pm 10.22 \mathrm{a}$ & 1.15 & 0.27 \\
Eh suelo $(\mathrm{mV})$ & $76.47 \pm 35.52 \mathrm{a}$ & $89.57 \pm 27.55 \mathrm{a}$ & 0.30 & 0.77 \\
Contenido de agua en suelo $(\%)$ & $69.95 \pm 1.22 \mathrm{a}$ & $66.90 \pm 1.62 \mathrm{a}$ & 1.76 & 0.11 \\
Densidad aparente del suelo $\left(\mathrm{g} \cdot \mathrm{cm}^{-3}\right)$ & $0.29 \pm 0.01 \mathrm{a}$ & $0.31 \pm 0.02 \mathrm{a}$ & 1.52 & 0.16 \\
\hline
\end{tabular}
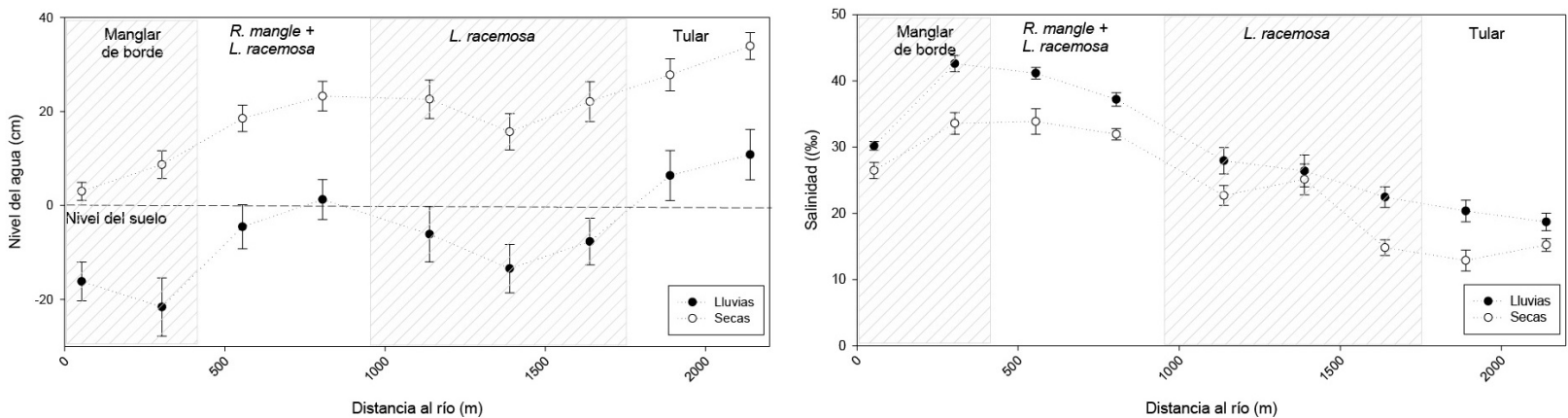

Figura 2. Variación espacial del nivel del agua (izquierda) y salinidad del agua intersticial (derecha) en relación con la distancia al río en meses de poca precipitación (diciembre-mayo) y meses con mayor precipitación (junio-noviembre). Se muestran los cuatro principales tipos de vegetación a lo largo del gradiente. Se presenta la media \pm 1 EE de 18 datos (6 meses por época x 3 transectos).

sobre el suelo se mantuvo en niveles bajos, a veces con aumentos intermitentes, pero de octubre a noviembre se produjo un aumento sostenido del nivel del agua hasta alcanzar los niveles más altos en diciembre, que es la temporada de nortes (Figura $3 \mathrm{~A}$ ). En el periodo 2015-2016 la diferencia entre el nivel más bajo y el más alto fue de $101.5 \mathrm{~cm}$; mientras que en el periodo 2018-2019 fue de $42.2 \mathrm{~cm}$.

\section{Distribución de la vegetación en el gradiente am- biental}

En la Figura 4 se presenta el clúster obtenido en el que se formaron tres grupos estadísticamente significativos (Prueba SIMPROF; $\mathrm{Pi}=6.22 ; \mathrm{P}=0.1 \%$; 999 iteraciones; sin iteraciones mayores o iguales al valor del estadígrafo $\mathrm{Pi}$ ). De izquierda a derecha de la figura del clúster, el primer grupo contiene cinco UM (cuatro del 2015 y una del 2019) caracterizadas por su composición herbácea dominada por T. domingensis, con presencia de $P$. australis y espacialmente se ubicaron en el extremo occidental del área de es- tudio. El segundo grupo sólo contiene a la UM-26 (dos UM, una de 2015 y la otra del 2019). Este grupo se separó de los demás porque la composición herbácea estuvo dominada por la gramínea Setaria sp., seguida en por la gramínea Sporobolus sp. El tercer grupo reportó el mayor número de UM y representa a la vegetación dominante del área de estudio que es de tipo arbórea, con mayor abundancia de $L$. racemosa, seguida por $R$. mangle, ambas especies en estadios juvenil y adulto. Este tercer grupo incluyó al subgrupo IIla en el que se ubicaron las UM cercanas al tular, donde $L$. racemosa crece en forma de arbusto y llega a mezclarse con $T$. domingensis. En el subgrupo IIlb estuvieron las UM con mayor abundancia de $L$. racemosa, tanto adultos como juveniles y ubicadas espacialmente a la mitad del gradiente manglar-tular. En el subgrupo IIlc hubo presencia importante de $R$. mangle. En el 2015 la UM 16 registró alta densidad de individuos juveniles de $L$. racemosa, pero en el 2019 hubo menor densidad, por lo que el análisis la dejó fuera del subgrupo Illb. La Figura 5 

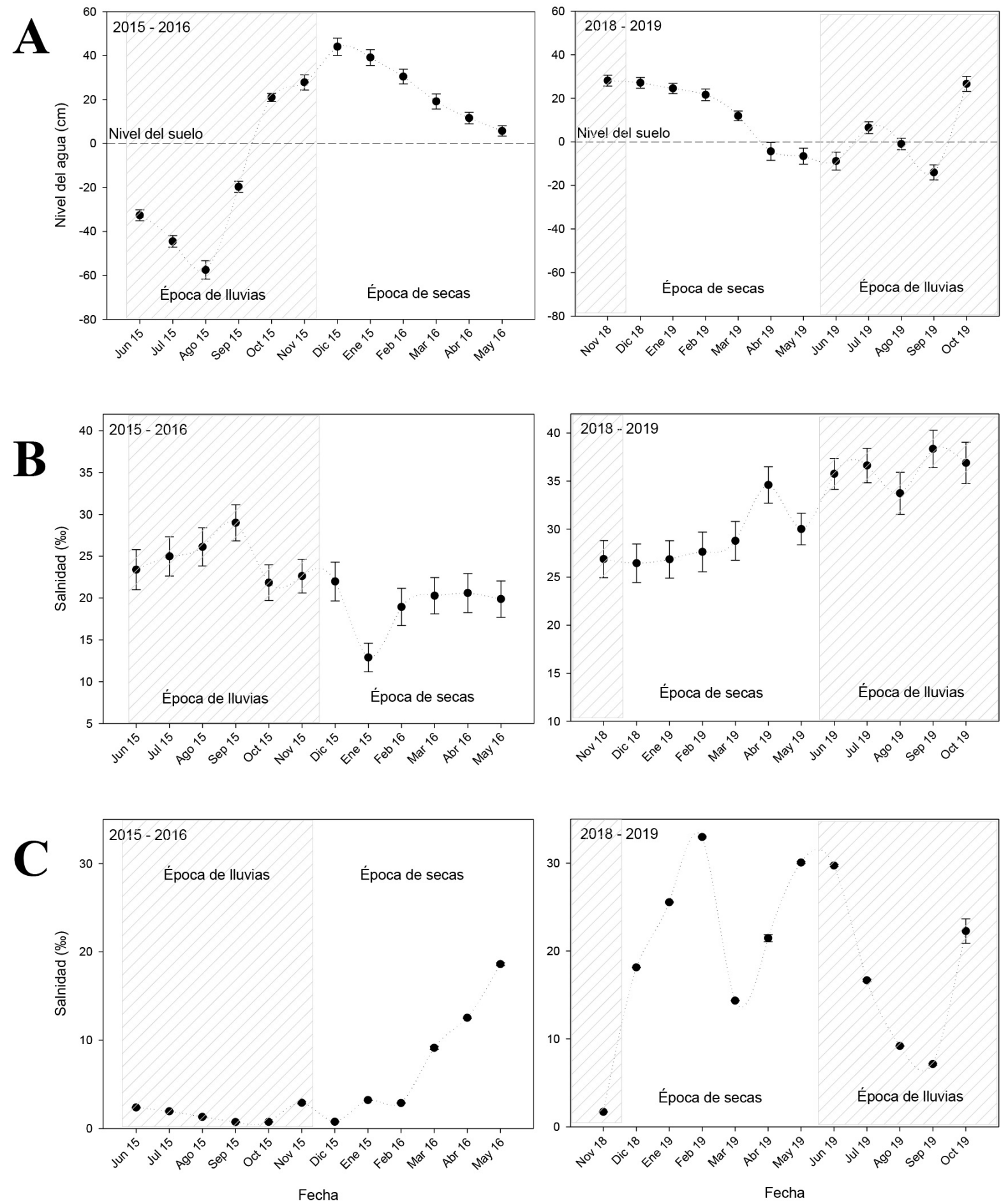

Figura 3. Los patrones temporales en el manglar-tular de Pantanos de Centla en los periodos del 2015-2016 y 2018-2019 se muestran a través de: A) Variación mensual (media \pm 1 EE de 27 Unidades de Monitoreo) del nivel del agua, B) Variación mensual (media $\pm 1 \mathrm{EE}$ de 27 Unidades de Monitoreo) de la salinidad del agua intersticial, C) Variación mensual (media \pm 1 EE de tres puntos cercanos al inicio de cada transecto) de la salinidad del agua superficial del río. 


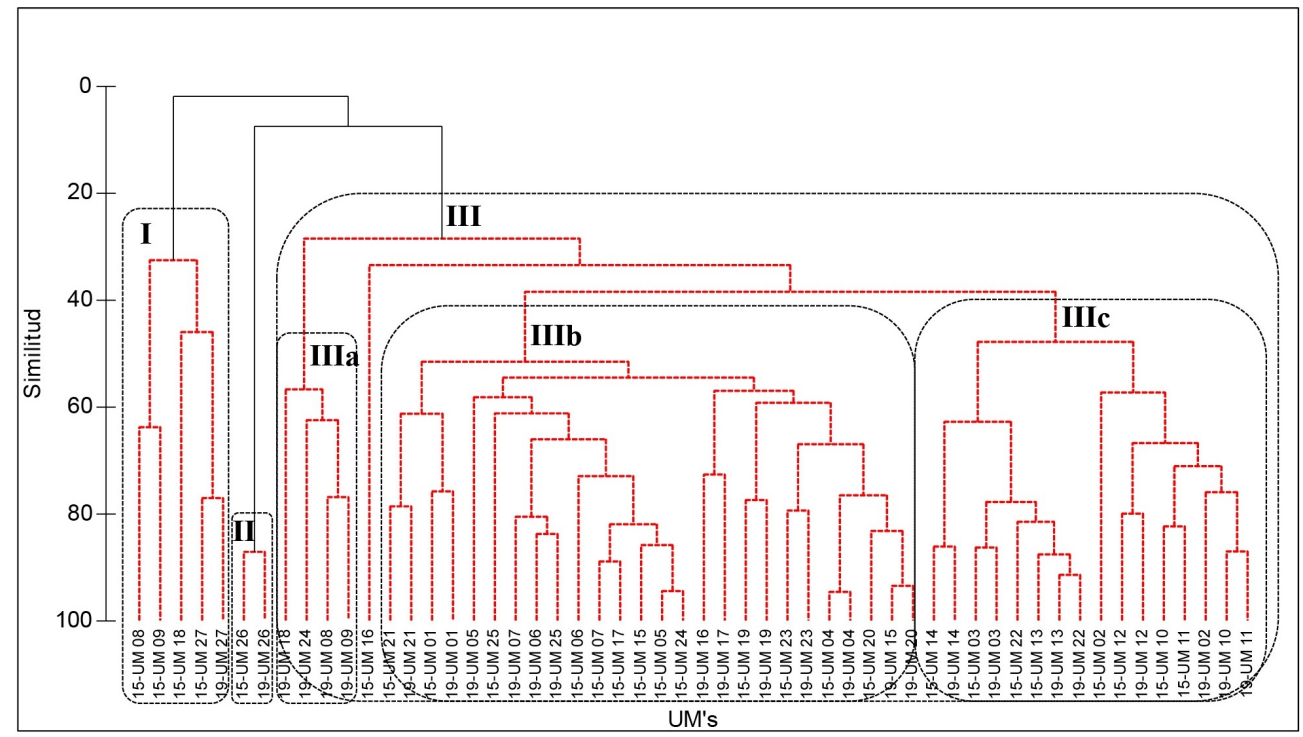

Figura 4. Agrupación numérica de 54 Unidades de Monitoreo (27 de 2015 y 27 de 2019) del manglar-tular de Pantanos de Centla.
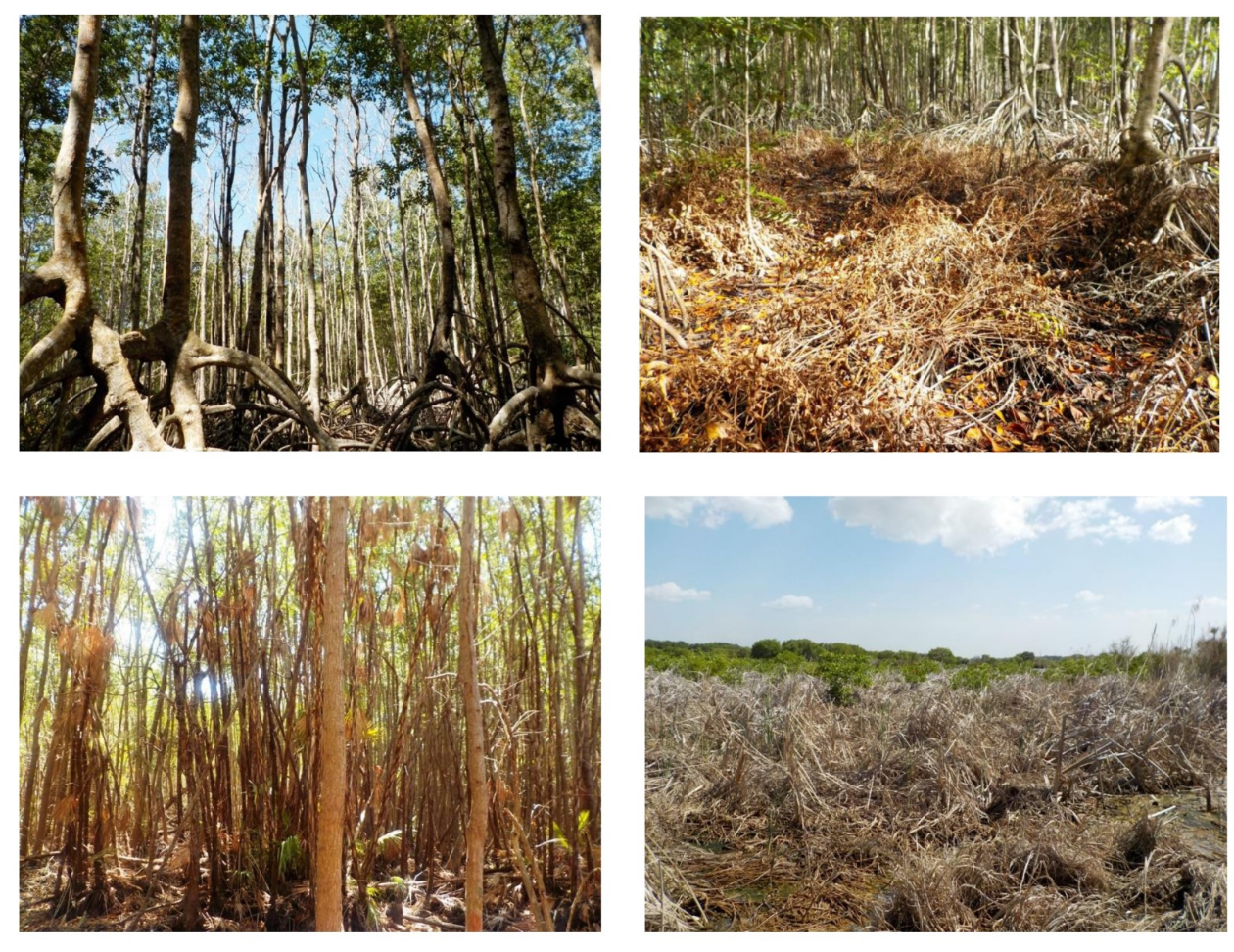

Figura 5. Fotografías del área de estudio en donde se muestra la formación de un claro de bosque de Rhizophora mangle (arriba a la izquierda, mayo de 2019), mortandad de Acrostichum danaeifolium (arriba a la derecha, abril de 2019), mortandad de parche de palmar de Acoelorraphe wrightii entre el bosque de Laguncularia racemosa (abajo a la izquierda, mayo de 2019), y mortandad de T. domingensis (abajo a la derecha, julio de 2019). 
muestra fotografías de los cambios observados. En el 2015, estas UM fueron dominadas por vegetación herbácea ( $T$. domingensis y $P$. australis); pero en el periodo 2018-2019 se detectó un incremento en la salinidad que provocó mortandad masiva de estas especies y un aumento en la presencia y abundancia de $L$. racemosa en forma de arbusto en las UM del extremo más alejado del río. En 2015 la UM 16 tuvo una baja densidad de individuos juveniles de L. racemosa, pero esta densidad aumentó en 2019; mientras que lo contrario ocurrió en la UM 24, en la que en 2019 murieron individuos juveniles de esta especie.

Con el análisis de gradiente directo BEST/BIOENV entre las matrices de similitud de las variables bióticas y abióticas se detectó que la vegetación respondió a un gradiente determinado por las variables nivel del agua, salinidad y $\mathrm{pH}$, así como por la ubicación espacial con respecto al río (latitud en UTM N y longitud en UTM E), obteniendo una correlación entre estas variables y la matriz de similitud (biótica) de 0.444 ( $R h o=0.444, \mathrm{P}=1 \%, 99$ permutaciones, sin permutaciones mayores o iguales al estadígrafo Rho obtenido).

\section{Conectividad hidrológica}

En la Figura 6 A y B se presenta la fluctuación de las dos variables relacionadas con la hidrología del manglar-tular del área de estudio (nivel del agua y salinidad), separando las UM más cercanas al río (manglar, UM 1, 2, 10, 11, 19 y 20) y las UM más alejadas (tular, UM 8, 9, 17, 18, 26 y 27). En ambos periodos de estudio la variación del nivel del agua (Figura 6 A) correspondió con la variación en los periodos de lluvias y secas. Los valores del nivel del agua encontrados en las UM del manglar fueron menores a los del tular (manglar $=-4.3 \pm 4.6 \mathrm{~cm}$; tular $=15.3 \pm 4.8 \mathrm{~cm} ; t_{\text {pareada }}=8.39 \mathrm{con} \mathrm{P}<0.001$ ), aunque en el segundo periodo ambos permanecieron más tiempo inundados que en el primer periodo.

La dinámica de la salinidad del agua intersticial (Figura 6 B) se mantuvo relativamente estable en el periodo 2015-2016 y entre noviembre 2018 y marzo 2019. Después de abril de 2019 se detectó un incremento en la salinidad en las comunidades de manglar y de tular. Durante ambos periodos de estudio, los valores de las UM localizadas en el manglar resultaron mayores a las del tular (manglar $=31.41 \pm$ 1.35 UPS; tular $=16.4 \pm 1.39$ UPS; $t_{\text {paread } a}=11.06$ con $\mathrm{P}<0.001$ ) y en este último, durante el segundo periodo, la salinidad de las siete UM se incrementó en una proporción promedio del $82 \%$ ( \pm 0.23\%).

\section{DISCUSIÓN}

En la literatura generalmente se describe al manglar como un ecosistema conectado con la laguna costera y los arrecifes (Du et al. 2020, Odériz et al. 2020), pero no con las selvas inundables (Davis et al. 2005, Ewel 2010) y los humedales herbáceos de agua dulce. En el programa "The Florida Coastal Everglades (FCE- LTER)" investigaron el sistema de humedales que incluyó el gradiente de manglar ribereño-humedal de agua dulce-pradera de pastos marinos (Troxler et al. 2015). En las planicies costeras mexicanas ya hay algunas descripciones del gradiente espacial de salinidad y de inundación desde el manglar hasta los humedales de agua dulce, similares al referido en el presente trabajo. FloresVerdugo et al. (2007) describieron este patrón de humedales para la costa de La Mancha, Veracruz. Moreno-Casasola et al. (2016) obtuvieron datos de la distribución espacial en relación con variables ambientales como la salinidad y el nivel de inundación en varias zonas costeras de Veracruz entre Tuxpan y Alvarado. Rincón-Pérez et al. (2020) detallaron los patrones de distribución del gradiente costero en EI Castaño, Chiapas. El presente trabajo aporta más información sobre la estrecha relación ecológica entre dos tipos de humedales que históricamente se han investigado por separado.

Durante el periodo de estudio no hubo cambios significativos en las variables de la estructura de la vegetación (densidad, área basal, altura), lo que podría indicar que el manglar de Pantanos de Centla es un ecosistema maduro, de dinámica estable, aunque se requiere un monitoreo de largo plazo para estimar la tendencia. En el espacio de dos kilómetros se produjeron cambios de vegetación, aunque la amplitud de los ámbitos de tolerancia a 

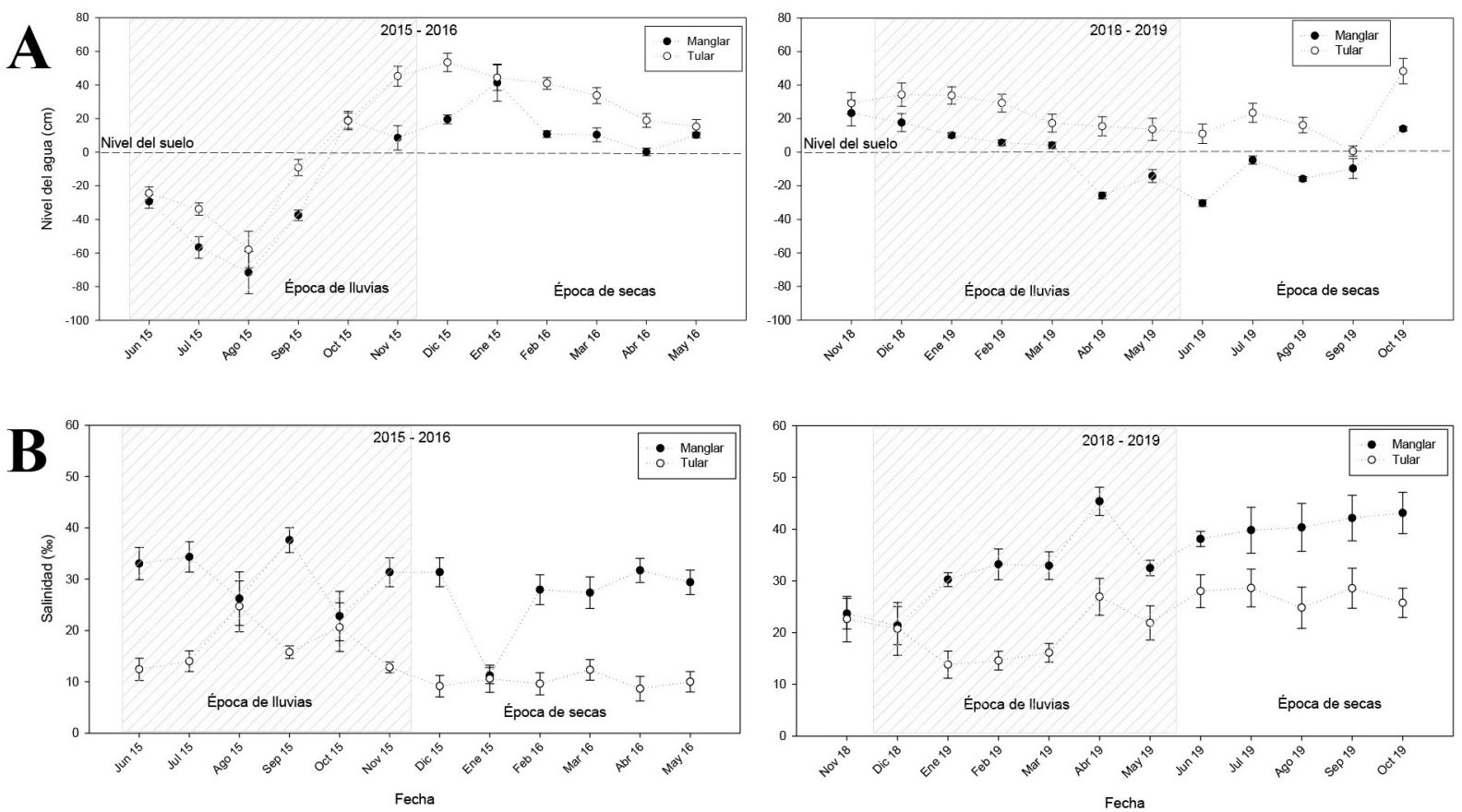

Figura 6. A) Variación temporal del nivel del agua en las Unidades de Monitoreo del manglar y del tular de Pantanos de Centla, en los periodos del 2015-2016 y 2018-2019. B) Variación temporal de la salinidad del agua intersticial en las Unidades de Monitoreo del manglar y del tular de Pantanos de Centla, en los periodos del 2015-2016 y 2018-2019.

la inundación y salinidad de las especies impidió que se encontraron diferencias significativas. Esta es una diferencia con respecto de las zonas antes mencionadas de Chiapas y Veracruz, donde hay selvas inundables de Pachira aquatica como parte del gradiente. En este estudio, en un espacio muy pequeño se produjo un cambio de un manglar mesohalino (salinidad del agua intersticial de 5 a 18 UPS) con presencia de palmas ( $A$. wrigthii) a un tular. Esto posiblemente se explica por los niveles de inundación que se mantienen casi todo el año en las zonas de tular y de manglar y en cambio hay mayores periodos de suelo que queda seco en la zona de mangle blanco y la palma $A$. wrightii. Ello indica una presencia importante de agua dulce continental que baja hacia la costa y mantiene estos niveles de inundación favoreciendo la presencia de humedales herbáceos. A pesar de que en Tabasco sí hay selvas inundables de $P$. aquatica (Maldonado-Sánchez et al. 2016), en la zona no se encontraron, posiblemente debido a que las selvas inundables se desarrollan en zonas de inundación temporal y por agua dulce.
En El Castaño (Chiapas), Rincón-Pérez et al. (2020) registraron el tiempo de mayor inundación en los dos extremos del gradiente, en el manglar y en el pastizal inundable. Los autores lo explicaron por el origen de las aportaciones de agua al sistema. En el pastizal, el humedal más alejado del mar, hay muy poca influencia marina. El agua que produce la inundación llega por la precipitación, el desbordamiento de los ríos y el agua subterránea proveniente de la sierra, por lo que permanece inundado durante la temporada de lluvias y parte de la temporada de secas ya que los aportes del agua subterránea continúan fluyendo; ello indica que esta es una zona de descarga de agua de la cuenca.

En el área de estudio el gradiente está dado principalmente por la salinidad. En El Castaño también está definido por la salinidad, de mayor a menor, principalmente del agua subterránea (Rincón-Pérez et al. 2020). Estos autores encontraron que en el caso del tular y la selva inundable la salinidad fue mayor a la reportada por Flores-Verdugo et al. (2007) y Moreno-Casasola et al. (2016) en algunos 
humedales en Veracruz y consideraron que esto se debe a la corta distancia en que se ubican los tulares y los remanentes de la selva inundable con respecto al mar. También consideran que durante la temporada de secas esto es más evidente, al presentar los mayores valores de salinidad, que llegan a estar por arriba de 15 UPS. Valores similares se encontraron para los tulares en el presente trabajo durante el primer periodo de medición; sin embargo, durante el segundo se duplicaron o triplicaron, siendo equivalentes a los reportados para el manglar.

Desde el punto de vista temporal se encontraron diferencias en el nivel de salinidad tanto del agua superficial del río como del agua intersticial, no así en el nivel de inundación. El aumento en la concentración de salinidad pudo deberse a un fenómeno de intrusión salina. Es una región donde hay una fuerte erosión costera (entre -2.32 y $-3.31 \mathrm{~m} \cdot \mathrm{año}^{-1}$; Núñez Gómez et al. 2016, Luijendij et al. 2018), por lo que la probabilidad de intrusiones salinas es cada vez mayor (Kuan et al. 2012). Por ser un humedal influido por la marea, pudo ocurrir intrusión salina de forma puntual por un fenómeno de marejada o mar de fondo. En el periodo de febrero a abril de 2019 hubo incidencia de frentes fríos que provocaron oleaje de más de dos metros de altura en la zona (CONAGUA 2019, SEMAR 2019); West et al. (1985) reportaron que la variación de las olas en la zona va de 0.3 a $0.7 \mathrm{~m}$ en las temporadas sin lluvia ni viento; pero en la época de nortes las olas exceden los cinco metros de altura (Lankford 1976). El oleaje de la temporada de nortes 2018-2019 pudo provocar el aumento de la salinidad y de nivel del agua reportados en el presente trabajo. El aumento en el nivel del agua y la salinidad de los humedales costeros por eventos puntuales de marejadas de tormenta será cada vez más frecuente como resultado del incremento en el nivel del mar (Craft et al. 2009, Karim y Mimura 2008, Hoffman et al. 2010). Éste ha sido medido y reportado en diferentes regiones del mundo, incluyendo el Golfo de México (Herbert et al. 2015, McCarthy et al. 2018, Zhai et al. 2018, van Dij et al. 2019, Wilson et al. 2019) y ha sido reconocido como una causa de aumento de salinidad en los humedales. Ramos-Reyes et al. $(2016 \mathrm{a}, \mathrm{b})$ concluyeron que las áreas protegi- das de Pantanos de Centla y Laguna de Términos están en la zona más vulnerable a erosión e inundaciones por el incremento en el nivel del mar. Gama Campillo et al. (2019) estimaron que más del $10 \%$ del territorio de Tabasco será afectado por un incremento de 0.5 a $1 \mathrm{~m}$ del nivel del mar hacia el 2100. Para cuantificar la magnitud de la intrusión salina por incremento en el nivel del mar y por efectos puntuales de marejada de tormenta en el área de estudio, es necesario un monitoreo integral de la hidrología con sensores automatizados de medición de nivel del agua y salinidad, de precipitación, evaporación, temperatura atmosférica, e instalación de piezómetros en distintas profundidades para detectar el origen del agua subterránea y la dirección del flujo (Sánchez-Cabeza et al. 2019, Sánchez Higueredo et al., 2020). Otras posibles causas del incremento de la salinidad en los humedales costeros son las sequías y la construcción de presas y otras estructuras que interrumpen el flujo de agua superficial a la cuenca baja, como los distritos de riegos en las cuencas media y alta (Gong y Shen 2011, Rivera-Monroy et al. 2011, Tully et al. 2019). Entre los trabajos que reportaron modificaciones en los distintos tipos de alteraciones a la hidrología en las cuencas Grijalva-Usumacinta se encuentran los de Castillo (2020), Cruz Ramírez et al. (2019), Medellín et al. (2013) y Muñoz-Salinas y Castillo (2005). En la cuenca del Usumacinta no hay presas, pero en el periodo del 2017-2019 hubo 513 $\mathrm{mm}$ menos de precipitación que en el periodo 20142016, en el que se presentó el Fenómeno El Niño (Yin et al. 2021).

El presente estudio de mediano plazo describió los cambios de vegetación y ambientales registrados a lo largo del gradiente, pero no permitió corroborar la causa del fenómeno y, como se mencionó, se requiere de un monitoreo continuo y de largo plazo para especificar la causa de la intrusión; o bien, comparar con resultados obtenidos en otras regiones del Golfo de México en el mismo periodo. El aumento rápido de salinidad genera estrés osmótico en organismos que crecen en agua dulce u oligohalinas (Marks et al. 2016). En las UM las especies más afectadas por la mayor salinidad fueron $T$. domingensis y $A$. danaeifolium. Aunque T. domingensis es 
una de las especies de humedales de agua dulce que toleran niveles bajos de salinidad (Glenn et al. 1995, Flores-Verdugo et al., 2007), no toleró los niveles alcanzados en esta área. Fuera de las UM también se observó la muerte de los pocos individuos de $A$. glabra y de parches completos de la palma $A$. wrigthii, así como formación de claros de bosque por mortandad reciente de árboles de $L$. racemosa. Este nivel de impacto indica que no es un fenómeno que se dé frecuentemente, independientemente de su origen y que está produciendo cambios en la composición y estructura de los humedales. Rivera-Monroy et al. (2011) reportaron avance del manglar hacia los humedales de agua dulce como efecto de la intrusión salina en los Everglades, resultado tanto del aumento en el nivel del mar como del manejo del agua dulce en la cuenca. Si el evento de incremento de salinidad fue derivado de marejada de tormenta, se espera que este fenómeno será cada vez más frecuente por el incremento en el nivel del mar y, por lo tanto, en el largo plazo el manglar seguirá avanzando dentro de lo que ahora es un humedal herbáceo.

A diferencia de lo que se ha obtenido en relación a la hidrología de otros humedales costeros del Golfo de México (López Rosas et al. 2006, Moreno-Casasola et al. 2010, 2017, Vázquez Benavides et al. 2020) o del Pacífico (Rincón Pérez et al. 2020), donde los niveles del agua se corresponden con la entrada de agua por la temporada de lluvias; en el presente trabajo se detectó un desfase entre los primeros meses de lluvia y el aumento en el nivel del agua; así como entre el inicio de la temporada de secas y la disminución del nivel del agua. Al inicio de la temporada de lluvias el suelo está muy seco, las lluvias no son abundantes y, por la cercanía al mar, es muy probable que el nivel del agua esté más influido por el nivel de las mareas, que actúan como una barrera al flujo, que por la entrada de agua de lluvia. En septiembre y octubre son los meses más lluviosos y los niveles de las mareas en la zona son más elevados (calendarios gráficos de mareas; SEMAR 2021), por lo que hay un aumento notable en el nivel del agua, provocando la inundación del área de estudio. En diciembre la cantidad de lluvia empieza a disminuir gradualmente en el tiempo, pero el nivel del agua se mantiene elevado porque el área está ubicada en la parte más baja de una cuenca hidrológica bastante extensa, y aunque la entrada por lluvia ya no es significativa, seguramente sí lo es la entrada subterránea (Euliss Jr. et al. 2004, Correa-Araneda et al. 2012, Lucas et al. 2014). Ello no ocurre en cuencas no tan extensas, como las del Pacífico o de otras regiones del Golfo de México. Este tipo de resultados apunta hacia la necesidad de realizar mayor cantidad de estudios sobre la geohidrología de los humedales de la planicie costera y su conectividad, así como monitoreo en el largo plazo, para poder entender la dinámica entre el agua dulce y la salina en el tiempo y el espacio en diferentes regiones del país, y poder predecir los impactos del cambio climático.

Recapitulando, en el plano espacial, conforme aumenta el nivel de inundación y disminuye la salinidad, las especies de mangle van disminuyendo en altura y su hábito cambia de arbóreo a arbustivo. Avicennia germinans sólo está presente en el extremo cercano al río, $R$. mangle es abundante en el extremo cercano al río, junto con $L$. racemosa, pero su abundancia disminuye gradualmente hasta que sólo domina un bosque monoespecífico de $L$. racemosa; continuando en el gradiente, ésta última especie sigue dominando, pero en forma arbustiva y en el extremo más alejado del río es reemplazada por vegetación herbácea dominada por $P$. australis y $T$. domingensis. En el plano temporal, en un periodo relativamente corto (2016 - 2019) se presentaron cambios en la composición y estructura de los humedales, debido a un incremento de la salinidad en general y a un cambio en los patrones de inundación, demostrando que estos humedales tienen una adaptación rápida y el sistema considerado como humedal, es resiliente, aunque con cambios en el tipo de humedal.

\section{CONCLUSIONES}

Por lo obtenido en la presente investigación podemos concluir que: (1) la vegetación del manglartular de Pantanos de Centla responde a un gradiente que se distribuye en función del nivel del agua que 
aumenta gradualmente conforme aumenta la distancia al río; (2) la salinidad del agua intersticial disminuye gradualmente en dirección del río (manglar) hacia tierra adentro (tular); el análisis del gradiente indirecto detectó una correlación significativa entre la distribución de la vegetación y la combinación de las variables salinidad de agua intersticial, distancia al río y $\mathrm{pH}$; (3) las asociaciones vegetales presentes en el área de estudio están conectadas hidrológicamente; esta conectividad está influida por las mareas, el nivel del río y las marejadas ciclónicas; (4) durante las mareas altas y las marejadas ciclónicas el nivel del agua aumenta en dirección mar-río, aumentando los niveles de inundación y salinidad en el sistema; este proceso se revierte en mareas bajas; (5) en época de lluvias, de septiembre a noviembre, ocurre el mayor aumento en el nivel del agua, respondiendo a un pulso de inundación del río y provocando una disminución en la salinidad en todo el sistema; (6) en el periodo del 2018-2019 se detectó un incremento en la salinidad intersticial en toda la zona de estudio, lo que afectó negativamente a especies con mayor afinidad a condiciones mesohalinas o dulces ( $T$. domingensis, $P$. australis, A. danaeifolium, A. glabra, Sporobolus sp. y A. wrigtii) y benefició a L. racemosa que empezó a aumentar su abundancia en la zona del tular. Los resultados obtenidos en el presente trabajo son representativos para los humedales riparios, influidos por la marea, asociados a la desembocadura del río Grijalva y del San Pedro San Pablo, de la Reserva de la Biósfera Pantanos de Centla, que son los humedales más cercanos a la costa. La conservación de los humedales costeros de esta Área Natural Protegida dependerá de conocer con certeza los fenómenos climáticos y antropogénicos implicados en los procesos que afectan a su vegetación, tales como la fluctuación del nivel del agua y la salinidad del agua intersticial.

\section{AGRADECIMIENTOS}

A la Comisión Nacional para el Conocimiento y Uso de la Biodiversidad (CONABIO) por el financiamiento de los proyectos LM001 y LM020. A Roberto Monroy por la elaboración del mapa del área de estudio. A los estudiantes de la Licenciatura en Biología Marina de la UNACAR por su apoyo voluntario en campo y laboratorio: Cinthia $F$. Arjona Jiménez, Yammilet D. Balboa Nieto, Claudia M. Bello de la Cruz, Pedro Castellano Pérez, Andrés Cruz Quintana, Johnny C. de la Cruz Dantorie, Monserrat Medina Acevedo, Kevin D. Méndez Martínez, Daniel A. Tovar Montalvo. A Citlalli G. Garrido Abreu y Thelma P. López García por su apoyo técnico.

\section{LITERATURA CITADA}

Barendregt A, Swarth CW (2013) Tidal freshwater wetlands: variation and changes. Estuaries and Coasts 3: 445-456.

Benítez Torres JA, Villalobos-Zapata GJ (2010) Sitios prioritarios para la conservación. In: Villalobos-Zapata GJ, Mendoza Vega J (Coord.) La Biodiversidad en Campeche: Estudio de Estado. CONABIO/ Gobierno del Estado de Campeche/Universidad Autónoma de Campeche/ECOSUR. México. pp: 586-606.

Bracken LJ, Croke J (2007) The concept of hydrological connectivity and its contribution to understanding runoffdominated geomorphic systems. Hydrological Processes 21: 1749-1763.

Brower JE, Zar JH, von Ende CN (1998) Field and Laboratory Methods for General Ecology. WCB-McGraw-Hill. Boston. 273p.

Castillo MM (2020) Suspended sediment, nutrients, and chlorophyll in tropical floodplain lakes with different patterns of hydrological connectivity. Limnologica 82: 125767.

Cintrón GA, Lugo E, Martínez R (1985) Structural and functional properties of mangrove forests. In: D’Arcy WG, Corma MD (eds.) The botany and natural history of Panama, IV Series: Monographs in Systematic Botany, 
Vol. 10. Missouri Botanical Garden. San Luis, Missouri. pp: 53-66.

Clarke KR (1993) Non-parametric multivariate analyses of changes in community structure. Australian Journal of Ecology 18: 117-143.

Clarke KR, Somerfield PJ, Gorley RN (2008) Testing of null hypotheses in exploratory community analyses similarity profiles and biota-environment linkage. Journal of Experimental Marine Biology 366: 56-69.

CONAGUA (2019) Pronóstico Meteorológico General. No. aviso: 125. Servicio Meteorológico Nacional, Comisión Nacional del Agua. Ciudad de México. 4p.

CONAGUA (2021) Normales Climatológicas por Estado (Benito Juárez, Centla, Tabasco). Comisión Nacional del Agua. https://smn.conagua.gob.mx/es/informacion-climatologica-por-estado?estado=tab. Fecha de consulta: 29 de abril, 2021.

Correa-Araneda FJ, Urrutia J, Soto-Mora Y, Figueroa R, Hauenstein E (2012) Effects of the hydroperiod on the vegetative and community structure of freshwater forested wetlands, Chile. Journal of Freshwater Ecology 27: 459-470.

Craft C, Clough J, Ehman J, Joye S, Park R, Pennings S, Guo H, Mschmuller M (2009) Forecasting the effects of accelerated sea-level rise on tidal marsh ecosystem services. Frontiers in Ecology and Environment 7 : 73-78.

Cruz-Ramírez AK, Salcedo MA, Sánchez AJ, Mendoza JD, Barba E, Álvarez-Pliego N, Florido F (2019) Intraannual variation of Chlorophyll-a and nutrients in a hydraulically perturbed wetland in the floodplain of the Grijalva river basin. Hidrobiológica 29: 163-170.

Davis S, Childers D, Lorenz J, Wanless H, Hopkins T (2005) A conceptual model of ecological interactions in the mangrove estuaries of the Florida Everglades. Wetlands 25: 832-842.

Day JW, Pont D, Hensel PF, Ibañez C (1995) Impacts of sea-level rise on deltas in the Gulf of Mexico and the Mediterranean: The importance of pulsing events to sustainability. Estuaries 18: 636-647.

Du J, Xie M, Wang Y, Chen Z, Liu W, Liao J, Chen B (2020) Connectivity of fish assemblages along the mangroveseagrass-coral reef continuum in Wenchang, China. Acta Oceanologica Sinica 39: 43-52.

Ellison A (2004) Wetlands of Central America. Wetland Ecology and Management 12: 3-55.

Euliss Jr. NH, LaBaugh JW, Fredrickson LH, Mushet DM, Laubhan MK, Swanson GA, Winter TC, Rosenberry DO, Nelson RD (2004) The wetland continuum: a conceptual framework for interpreting biological studies. Wetlands 24: 448-458.

Ewel KC (2010) Appreciating tropical coastal wetlands from a landscape perspective. Frontiers in Ecology and Environment 8: 20-26.

Flores-Verdugo F, Moreno-Casasola P, Agraz-Hernández CM, López Rosas H, Benítez Pardo D, Travieso-Bello AC (2007) La topografía y el hidroperíodo: dos factores que condicionan la restauración de los humedales costeros. Boletín de la Sociedad Botánica de México 80 (suplemento): 33-47.

Gama Campillo L, Macías-Valadez ME, Pacheco-Figueroa CJ, Collado-Torres RA, Mata-Zayas E, Díaz-López HM, Valdez Leal JD, Arriaga Weiss S, Rangel-Ruíz LJ. (2019) Capítulo 21. Panarquia: propuesta de análisis de la dinámica costera de Tabasco ante el cambio global. In: Rivera-Arriaga E, Azuz-Adeath I, Cervantes Rosas OD, Espinoza-Tenorio A, Silva-Casarín R, Ortega-Rubio A, Botello AV y Vega Serratos BE (Eds.) Gobernanza y manejo de las costas y mares ante la incertidumbre. Una guía para tomadores de decisiones. Universidad Autónoma de Campeche/RICOMAR. San Francisco de Campeche. pp: 427-447. 
Glenn E, Thompson TL, Frye R, Riley J, Baumgartner D (1995) Effects of salinity on growth and evapotranspiration of Typha domingensis Pers. Aquatic Botany 52: 75-91.

Gong W, Sheng J (2011) The response of salt intrusion to changes in river discharge and tidal mixing during the dry season in the Modaomen Estuary, China. Continental Shelf Research 31: 769-788.

González-Marín, RM, Moreno-Casasola P, Orellana R, Castillo A (2012) Palm use and social values in rural communities on the coastal plains of Veracruz, Mexico. Environment, Development and Sustainability 14: 541-555.

Guerra-Martínez V, Ochoa-Gaona S (2018). Evaluación del programa de manejo de la Reserva de la Biosfera Pantanos de Centla en Tabasco, México. Universidad y Ciencia 24: 135-146.

Herbert ER, Boon P, Burgin AJ, Neubauer SC, Franklin RB, Ardón M, Hopfensperger KN, Lamers LPM, Gell $P$ (2015) A global perspective on wetlands salinization: Ecological consequences of a growing threat to freshwater wetlands. Ecosphere 6: 1-43.

Herrera Silveira JA, Camacho Rico A, Pech E, Pech M, Ramírez Ramírez J, Teutli Hernández C (2016) Dinámica del carbono (almacenes y flujos) en manglares de México. Terra Latinoamericana 34(1): 61-72.

Hoffman RN, Dailey P, Hopsch S, Ponte RM, Quinn K, Hill EM, Zachry B (2010) An estimate of increases in storm surge risk to property from sea level rise in the first half of the twenty-first century. Weather, Climate, and Society 2(4): 271-293.

Infante-Mata D, Moreno-Casasola P, Madero-Vega C (2014) Pachira aquatica, as indicator of mangrove limit? Revista Mexicana de Biodiversidad 85: 143-160.

Karim MF, Mimura N (2008) Impacts of climate change and sea-level rise on cyclonic storm surge floods in Bangladesh. Global Environmental Change 18: 490-500.

Kent M (2011) Vegetation Description and Data Analysis: A Practical Approach, $2^{a}$ Edición. Wiley-Blackwell, Londres. 428p.

Kuan WK, Jin G, Xin P, Robinson C, Bibbes B, Li L (2012) Tidal influence on seawater intrusion in unconfined coastal aquifers. Water Resources Research 48: 1-11.

Landgrave R, Moreno-Casasola P (2012) Evaluación cuantitativa de la pérdida de humedales en México. Investigación Ambiental 4: 19-35.

Lankford RR (1976) Coastal lagoons of Mexico: their origin and classification. In: Wiley M (Ed.) Estuarine processes. Academic Press. Nueva York. pp: 182-215.

Legendre P, Legendre L (1998) Numerical ecology. $2^{a}$ Edición. Elsevier. Nueva York. 853p.

López Portillo J, Ezcurra E (2002) Los manglares de México: una revisión. Madera y Bosques Número Especial: 27-51.

López Rosas H, Moreno-Casasola P, Mendelssohn IA (2006) Effects of experimental disturbances on a tropical freshwater marsh invaded by the African grass Echinochloa pyramidalis. Wetlands 26: 593-604.

López Rosas H, Tolome Romero J (2009) Medición del potencial redox del suelo y construcción de electrodos de platino. In: Moreno-Casasola P, Warner B (eds.) Breviario para describir, observar y manejar humedales. Serie costa sustentable no 1. RAMSAR/INECOL/CONANP/US Fish and Wildlife Service/US State Department. Xalapa. pp: 131-138.

Lot-Helgueras A, Novelo A (1990) Forested Wetlands of Mexico. In: Lugo AE, Brinson MM, Brown S (eds.) Ecosystems of the world. Forested wetlands of the world. Elsevier. Amsterdam. pp: 287-298. 
Lucas CM, Schöngart J, Sheikh P, Wittmann F, Piedaded MTF, McGrath DG (2014) Effects of land-use and hydroperiod on aboveground biomass and productivity of secondary Amazonian floodplain forests. Forest Ecology and Management 319: 116-127.

Luijendijk A, Hagenaars G, Ranasinghe R, Baart F, Donchyst G, Aarninkhof S (2018) The state of the world's beaches. Scientific Report 8: 6641.

Maldonado-Sánchez EA, Ochoa-Gaona S, Ramos-Reyes R, Guadarrama-Olivera MÁ, González-Valdivia N, Jong BHJ (2016). La selva inundable de canacoite en Tabasco, México, una comunidad vegetal amenazada. Acta Botánica Mexicana 115: 75-101.

Marks BM, Chambers L, White JR (2016) Effect of fluctuating salinity on potential denitrification in coastal wetland soil and sediments. Soil Science Society of America Journal 80: 516-526.

McCarthy MJ, Dimmitt B, Muller-Karger FE (2018) Rapid coastal forest decline in Florida's Big Bend. Remote Sensing 10: 1721.

McKee KL (2012). Neotropical coastal wetlands. In: Batzer DP, Baldwin AH (eds.) Wetland habitats of North America: Ecology and Conservation Concerns. University of California Press. Berkeley. pp: 89-101.

Medellín G, Mariño-Tapia I, Pedrozo-Acuña A, Enriquez C, González-Villareal F (2013) Influencia de la marea en la hidrodinámica del sistema Mecoacán-Río González, México. Tecnología y Ciencias del Agua 4: 71-90.

Mitsch WJ, Gosselink JG (2007) Wetlands, J Wiley \& Sons Inc., Hoboken, Nueva Jersey. 582p.

Moreno-Casasola P (2008) Los humedales en México: tendencias y oportunidades. Cuadernos de Biodiversidad 28: $10-18$.

Moreno-Casasola P, Cejudo E, Monroy R, Infante Mata D, López Rosas H, Peralta Peláez LA, Neri Flores I, Castillo-Campos G, Madero Vega C, Capistrán A, Rincón M, Pérez Torres S (2016) Manglares, selvas inundables y humedales herbáceos. In: Moreno-Casasola P (ed.) Servicios Ecosistémicos de Selvas y Bosques Costeros de Veracruz. Costa Sustentable no 8. INECOL/ITTO/CONAFOR/INECC. Xalapa. pp: 76-94.

Moreno-Casasola P, Cejudo-Espinosa E, Capistrán Barradas A, Infante-Mata D, López-Rosas H, Castillo-Campos G, Pale-Pale J, Campos-Cascaredo A (2010) Composición florística, diversidad y ecología de humedales herbáceos emergentes en la Planicie Costera Central de Veracruz, México. Boletín de la Sociedad Botánica de México 87: 29-50.

Moreno-Casasola P, Hernández ME, Campos A (2017) Hydrology, soil carbon sequestration and water retention along a coastal wetland gradient in the Alvarado lagoon system, Veracruz, Mexico. Journal of Coastal Research 77: 104-115.

Moreno-Casasola P, Infante Mata D, López Rosas H (2012) Tropical freshwater swamps and marshes. En: Batzer DP, Baldwin AH (eds.) Wetland habitats of North America: Ecology and conservation concerns. University of California Press, Berkeley. pp: 267-282.

Muñoz-Salinas E, Castillo M (2015) Streamflow and sediment load assessment from 1950 to 2006 in the Usumacinta and Grijalva Rivers (Southern Mexico) and the influence of ENSO. CATENA 127: 270-278.

Novelo A, Ramos L (2005) Vegetación acuática. In: Bueno J, Álvarez F, Santiago S (eds.) Biodiversidad del Estado de Tabasco. Instituto de Biología, UNAM/CONABIO. México. pp: 111-144.

Novelo-Retana A (2006) Plantas Acuáticas de la Reserva de la Biosfera Pantanos de Centla. CONABIO/CONANP/ Espacios Naturales y Desarrollo Sustentable A.C./Instituto de Biología, UNAM/PEMEX, México. 260p. 
Núñez Gómez JC, Ramos Reyes R, Barba Macías E, Espinoza Tenorio A, Gama Campillo LM (2016) Índice de vulnerabilidad costera del litoral tabasqueño, México. Investigaciones Geográficas 91: 70-85.

Nyman JA, Delaune RD, Patrick WH (1990) Wetland soil formation in the rapidly subsiding Mississippi River deltaic plain-mineral and organic-matter relationships. Estuarine Coastal Shelf Science 31: 57-69.

Odériz I, Gómez I, Ventura Y, Díaz V, Escalante A, Gómez DT, Bouma TJ, Silva R (2020) Understanding drivers of connectivity and resilience under tropical cyclones in coastal ecosystems at Puerto Morelos, Mexico. Journal of Coastal Research 95: 128-132.

Olmsted I (1993) Wetlands of Mexico. In: Whigham DF, Dykyjová D, Hejný S (eds.) Wetlands of the World I: Inventory, Ecology and Management. Kluwer Academic Publishers. Dordrecht, Países Bajos. pp: 637-677.

Peralta Peláez LA, Infante Mata DM, Moreno-Casasola P (2009) Construcción e instalación de piezómetros. In: Moreno-Casasola P, Warner B (eds.) Breviario para Describir, Observar y Manejar Humedales. Serie Costa Sustentable no 1. RAMSAR/INECOL/CONANP/US Fish and Wildlife Service/US State Department. Xalapa. pp: 17-30.

Ramos Reyes R, Gama Campillo LM, Núñez Gómez JC, Sánchez Hernández R, Hernández Trejo H, Ruíz Álvarez O (2016a) Adaptación del modelo de vulnerabilidad costera en el litoral tabasqueño ante el cambio climático. Revista Mexicana de Ciencias Agrícolas 13: 2551-2563.

Ramos-Reyes R, Zavala-Cruz J, Gama-Campillo LM, Pech-Pool D, Ortiz-Pérez MA (2016b) Indicadores geomorfológicos para evaluar la vulnerabilidad por inundación ante el ascenso del nivel del mar debido al cambio climático en la costa de Tabasco y Campeche, México. Boletín de la Sociedad Geológica Mexicana 68(3): 581-598.

Ratti JT, Garton EO (1996) Research and experimental design. In: Bookhout TA (ed.) Research and Management Techniques for Wildlife and Habitats. The Wildlife Society. Maryland. pp: 1-23.

Rincón Pérez M, Infante-Mata D, Moreno-Casasola P, Hernández Alarcón ME, Barbas Macías E, García-Alfaro JR (2020) Patrones de distribución y estructura de la vegetación en el gradiente de humedales costeros El Castaño, Chiapas, México. Revista de Biología Tropical 68(1): 242-259.

Rivera-Monroy VH, Twilley RR, Davis SE, Childers DL, Simard M, Chambers R, Jaffe R, Boyer JN, Rudnick DT, Zhang K, Castañeda-Moya E, Ewe SML, Price RM, Coronado-Molina C, Ross M, Smith TJ, Michot B, Meselhe E, Nuttle W, Troxler TG, Noe GB (2011) The role of the Everglades mangrove ecotone region $(E M E R)$ in regulating nutrient cycling and wetland productivity in South Florida. Critical Reviews in Environmental Science and Technology 41: 633-669.

Rosas Urióstegui FI, Pat Fernández JM. Pat Fernández LA, van der Wal JC (2018) The effect of oil palm on income strategies and food security of households in rural communities in Campeche, Mexico. Acta Universitaria 28(3): 25-32.

Sánchez-Cabeza J-A, Álvarez Sánchez LF, Cardoso-Mohedano JG, Escalante Mancera E, Díaz-Asencio M, López-Rosas H, Machain-Castillo ML, Merino-lbarra M, Ruíz-Fernández AC, Alonso-Rodríguez R, GómezPonce MA, Ávila-Torres E, Rico-Esenaro S, Gómez-Reali MÁ, Herrera-Becerril CA, Solórzano-Ramos DE, Grutter M (2019) A low-cost long-term model of coastal observatories of global change. Journal of Operational Oceanography (12) 34-46.

Sánchez-Higueredo LE, Ramos-Leal JA, Morán-Ramírez J, Moreno-Casasola P, Rodríguez-Robles U, Hernández ME (2020) Ecohydrogeochemical functioning of coastal freshwater herbaceous wetlands in the Protected Natural Area, Ciénaga del Fuerte (American tropics): Spatiotemporal behaviour. Ecohydrology 13: e2173. DOI: $10.1002 /$ eco.2173 
SEMAR (2019) Resumen Anual de Frentes Fríos que Afectaron Aguas y Costas Mexicanas. 2018-2019. Secretaría de Marina Armada de México. Ciudad de México. 47p.

SEMAR (2021) Estación Mareográfica de Frontera, Tab. Secretaría de Marina. https://oceanografia.semar.gob. mx/Templates/grafnum_frontera.html. Fecha de consulta: 29 de abril, 2021.

Troxler TG, Gaiser E, Barr J, Fuentes JD, Jaffé R, Childers DL, Collado-Vides L, Rivera-Monroy VH, CastañedaMoya E, Anderson W, Chambers R, Chen M, Coronado-Molina C, Davis SE, Engel V, Fitz C, Fourqurean J, Frankovich T, Kominoski J, Madden C, Malone SL, Oberbauer SF, Olivas P, Richards J, Saunders C, Schedlbauer J, Scinto LJ, Sklar F, Smith T, Smoak JM, Starr G, Twilley RR, Whelan K (2015) Integrated carbon budget models for the Everglades terrestrial-coastal-oceanic gradient: Current status and needs for inter-site comparisons. Oceanography 26: 98-107.

Tully K, Gedan K, Epanchin-Niell R, Strong A, Bernhardt ES, BenDor T, Mitchell M, Kominoski J, Jordan TE, Neubauer SC, Weston NB (2019) The invisible flood: The chemistry, ecology, and social implications of coastal saltwater intrusion. BioScience 69: 368-378.

Valdez Hernández Jl (2002) Aprovechamiento forestal de manglares en el estado de Nayarit, costa Pacífica de México. Maderas y Bosques 8 (Número especial 1): 129-145.

van Dijk G, Lamers LPM, Loeb R, Westendorp P-J, Kuiperij R, van Kleef HH, Klinge M, Smolders AJP (2019) Salinization lowers nutrient availability in formerly brackish freshwater wetlands; unexpected results from a long-term field experiment. Biogeochemistry 143: 67-83.

Vázquez- Benavides J, López Rosas H, Moreno-Casasola P (2020) Effect of the grass Leersia hexandra on the dispersal, seed germination and establishment of Pachira aquatica seedlings. Freshwater Biology 65: $1702-1717$.

West R, Psuty NP, Thom B (1985) Las tierras bajas de Tabasco en el sureste de México. Instituto de Cultura de Tabasco. Villahermosa. 416p.

Wilson BJ, Serais S, Charles SP, Mazzei V, Gaiser E, Kominoski JS, Richards JH, Troxler TG (2019) Phosphorus alleviation of salinity stress: effects of saltwater intrusion on an Everglades freshwater peat marsh. Ecology 100(5): e02672. DOI: 10.1002/ecy.2672.

Yin J, Xu J, Xue Y, Xu B, Zhang C, Li Y, Ren Y (2021) Evaluating the impacts of El Niño events on a marine bay ecosystem based on selected ecological network indicators. Science of the Total Environment 763: 144205. DOI: 10.1016/j.scitotenv.2020.144205.

Zalizniak L, Kefford B, Nugegoda D (2009) Effects of pH on salinity tolerance of selected freshwater invertebrates. Aquatic Ecology 43: 135-144.

Zar JH (2009) Biostatistical Analysis. 5a Edición. Prentice Hall. Nueva Jersey. 666p.

Zhai L, Krauss KW, Liu X, Duberstein JA, Duberstein JA, Conner W, DeAngelis DL, Sternberg LdSL (2018) Growth stress response to sea level rise in species with contrasting functional traits: A case study in tidal freshwater forested wetlands. Environmental and Experimental Botany 155: 378-386.

Zhang Y, Li W, Sun G, King JS (2019) Coastal wetland resilience to climate variability: A hydrologic perspective. Journal of Hydrology 568: 275-284. 